\title{
ORIGINAL
}

\author{
T. Kaiser(D) - A. Menzel(D)
}

\section{An electro-mechanically coupled computational multiscale formulation for electrical conductors}

Received: 11 March 2020 / Accepted: 31 October 2020 / Published online: 16 December 2020

(C) The Author(s) 2020

\begin{abstract}
Motivated by experimental findings on deformation induced microcracks in thin metal films and by their influence on the effective macroscopic electrical conductivity, a computational multiscale formulation for electrical conductors is proposed in this contribution. In particular, averaging theorems for kinematic quantities and for their energetic duals are discussed, an extended version of the Hill-Mandel energy equivalence condition is proposed and suitable boundary conditions for the microscale problem are elaborated. The implementation of the proposed framework in a two-scale finite element environment is shown and representative boundary value problems are studied in two- and three-dimensional settings.
\end{abstract}

Keywords Flexible electronic devices - Electro-mechanical coupling - Conductors - Anisotropic conductivity $\cdot$ Heterogeneous microstructures $\cdot$ Multiscale modelling $\cdot$ Scale-bridging

\section{Introduction}

Advances in material science and fabrication technologies enable the development of flexible electronic devices like wearable sensors $[1,22]$ and foldable displays $[3,15]$, which are in the focus of many engineering applications. To ensure the functionality of these devices, detailed knowledge is required on the electro-mechanical material properties for various loading conditions. However, the experimental characterization of material thin films, especially the study of failure mechanisms, is difficult and requires the development of advanced measuring technologies as discussed in [4]. Computational multiscale homogenization schemes are promising numerical approaches to support these developments as they allow material microstructures and their evolutions to be resolved in numerical simulations. On the other hand, complex deformation processes which are difficult to study experimentally are accessible using suitable numerical multiscale formulations that were calibrated on the basis of experiments.

More specifically speaking, processes at different material length- and timescales are considered in finite element-based computational multiscale simulations with the evaluation of classic constitutive material models being replaced by finite element calculations of the underlying material microstructures. The latter ones are represented by representative volume elements (RVEs) that take distinct microstructural features like grains and microcracks into account $[9,27]$. The boundary conditions on the microscale are prescribed based on the macroscopic material state. Vice versa, effective energetic duals to the kinematic quantities on the macroscale

T. Kaiser $(\bowtie) \cdot$ A. Menzel

Institute of Mechanics, TU Dortmund University, Leonhard-Euler-Str. 5, 44227 Dortmund, Germany

E-mail: tobias.kaiser@tu-dortmund.de

E-mail: andreas.menzel@udo.edu

\author{
A. Menzel \\ Division of Solid Mechanics, Lund University, P.O. Box 118, 22100 Lund, Sweden \\ E-mail: andreas.menzel@solid.lth.se
}


are calculated based on their microscopic counterparts by using computational homogenization schemes. First-order computational multiscale formulations for mechanical problems have in the meanwhile been wellestablished and are for example discussed in [10,11,16,19-21]. Homogenization methods for generalized continua, in particular, second-order homogenization methods that account for the macroscopic deformation tensor and for its gradient, are elaborated in $[12,17,18,23]$. Moreover, extensions to thermomechanical coupling are presented in $[2,6,24,28,29]$ and electro-mechanically coupled multiscale formulations for piezo- and ferroelectric materials are discussed in $[13,14,26]$.

The present contribution establishes an electro-mechanically coupled computational multiscale framework for electrical conductors. In contrast to the theoretical developments on (dielectric) electro-active solids discussed in $[13,26]$, the electro-mechanical coupling in the electrical conductors considered is rather implicit. In particular, changes in electrical conductivity due to mechanically induced microcracks motivate the present contribution. Against this background, assume for now a homogeneous, quasi-one-dimensional electrical problem for which the effective macroscopic electrical resistance is given by

$$
R=\frac{1}{\kappa} \frac{L}{A}=R_{\text {spe }} R_{\text {geo }}, \quad R_{\text {spe }}=\frac{1}{\kappa}, \quad R_{\text {geo }}=\frac{L}{A} .
$$

Whereas the geometrical contribution $R_{\text {geo }}$ to the electrical resistance is defined by the macroscopic length $L$ and cross-sectional area $A$ of the conductor, the specific resistance $R_{\text {spe }}$ which is defined as the inverse conductivity $\kappa$ is an effective macroscopic material parameter that accounts for the (possibly inhomogeneous) material microstructure. The different contributions to the effective macroscopic resistance are exemplified in Fig. 1 for a material featuring microstructural imperfections. Furthermore, the influence of plastic deformation and of grain boundaries on the electrical conductivity may be taken into account. With regard to the influence of the electrical field quantities on the mechanical field quantities, it is observed that the dissipation associated with the electric current contributes to the balance equation of energy and that the induced changes in temperature, in turn, influence the mechanical properties. Finally, mechanically induced deformations directly influence the geometric part of the electrical resistance in finite deformation processes.

The article is organized as follows: After a brief summary of the governing field equations for electromechanical problems in Sect. 2, Sect. 3 focuses on the electro-mechanically coupled multiscale formulation. In particular, averaging theorems for kinematic quantities and for their energetic duals are elaborated, generalized Hill-Mandel conditions are derived and suitable boundary conditions are discussed. Based on these developments, a finite element implementation of the proposed theory is presented in Sect. 4. A study of representative boundary value problems in Sect. 5 eventually shows the applicability of the proposed formulation.

\subsection{Notation}

Let $\boldsymbol{\alpha}, \boldsymbol{\beta}, \boldsymbol{\gamma}$ and $\boldsymbol{\delta}$ denote arbitrary first-order tensors, let the standard dyadic product be indicated by $\otimes$ and let single tensor contractions be given by $[\boldsymbol{\alpha} \otimes \boldsymbol{\beta}] \cdot[\boldsymbol{\gamma} \otimes \delta]=[\boldsymbol{\beta} \cdot \boldsymbol{\gamma}][\boldsymbol{\alpha} \otimes \delta]$. Based on the latter definitions, the generalized dyadic products $[\boldsymbol{\alpha} \otimes \boldsymbol{\beta}] \otimes[\boldsymbol{\gamma} \otimes \delta]=[\boldsymbol{\alpha} \otimes \boldsymbol{\gamma}] \otimes[\boldsymbol{\beta} \otimes \delta]$ and $[\boldsymbol{\alpha} \otimes \boldsymbol{\beta}] \otimes[\boldsymbol{\gamma} \otimes \delta]=$ $[\boldsymbol{\alpha} \otimes \gamma] \otimes[\delta \otimes \beta]$ are introduced to shorten notation. Moreover, these serve as the basis for the introduction of higher-order identity tensors based on suitable combinations of second-order identity tensors $\boldsymbol{I}$. Double tensor contractions are defined as $[\boldsymbol{\alpha} \otimes \boldsymbol{\beta}]:[\boldsymbol{\gamma} \otimes \delta]=[\boldsymbol{\alpha} \cdot \boldsymbol{\gamma}][\boldsymbol{\beta} \cdot \boldsymbol{\delta}]$ and gradient, divergence and curl operators,
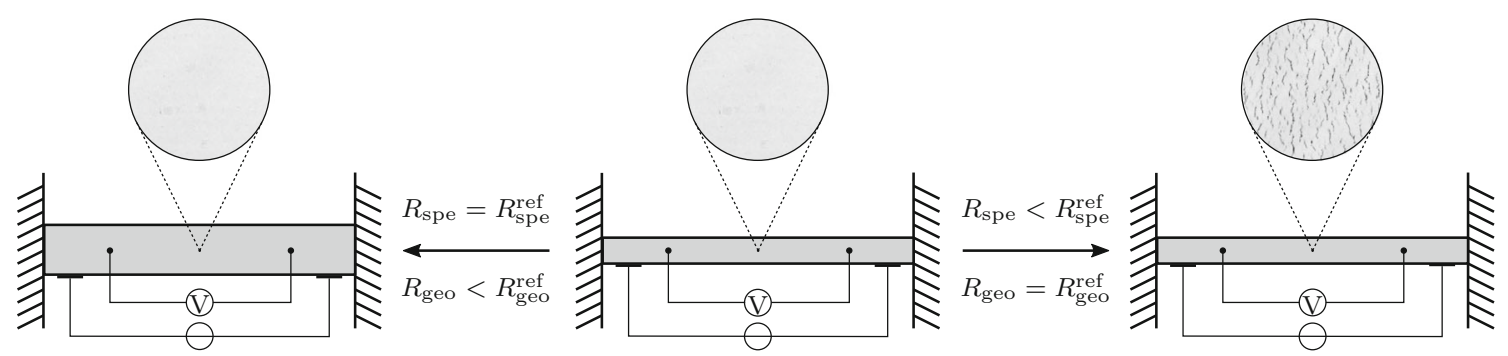

Fig. 1 Differences in the geometric dimensions and in the underlying microstructures of the idealized wire-like structures (left, right) manifest themselves in different geometric and specific resistances compared to the reference specimen (middle). Microstructure images courtesy of Megan J. Cordill, Erich Schmid Institute of Materials Science, Austrian Academy of Sciences 
indicated by $\nabla \bullet$, by $\nabla \cdot \bullet$ and by $\nabla \times \bullet$, respectively, are used in sense of right-gradient, right-divergence and right-curl operations.

\section{Continuum thermodynamics}

This section provides a summary of the thermodynamic fundamentals of the (thermo-)electro-mechanically coupled multi-field formulation. To this end, let the region that is occupied by the body under consideration at some reference time $t_{0} \in \mathbb{R}$ be denoted by $\mathcal{B}_{0} \subset \mathbb{R}^{3}$. Moreover, define the deformation field as the point mapping $\boldsymbol{\varphi}(\boldsymbol{X}, t): \mathcal{B}_{0} \times \mathbb{R} \rightarrow \mathcal{B}_{t} \subset \mathbb{R}^{3}$ that relates the referential position of a particle $\boldsymbol{X} \in \mathcal{B}_{0}$ to its counterpart $\boldsymbol{x} \in \mathcal{B}_{t}$ in the current configuration $\mathcal{B}_{t} \subset \mathbb{R}^{3}$ at time $t \geq t_{0}$. As is customary for infinitesimal deformation processes which are assumed in the following, the displacement field $\boldsymbol{u}=\boldsymbol{\varphi}(X, t)-X$ is additionally introduced and the reference and current configuration will not be distinguished, e.g., when defining integrals or derivatives, such that the respective superscripts are omitted in the following.

\subsection{Mechanical field equations}

Considering mass to be conserved, the mechanical response is governed by the balance equation of linear momentum

$$
\frac{\mathrm{d}}{\mathrm{d} t} \int_{\mathcal{B}} \rho \dot{\boldsymbol{u}} \mathrm{d} v=\int_{\mathcal{B}} \rho \boldsymbol{f} \mathrm{d} v+\int_{\partial \mathcal{B}} \boldsymbol{t} \mathrm{d} a
$$

with the mass density per unit volume $\rho$, the velocity vector $\dot{\boldsymbol{u}}$ and the body force density (per unit mass) $\boldsymbol{f}$. By invoking Cauchy's theorem to relate the stress vector $\boldsymbol{t}$ to the stress tensor $\boldsymbol{\sigma}$ and to the outward unit surface normal $\boldsymbol{n}$ according to

$$
\boldsymbol{t}=\boldsymbol{\sigma} \cdot \boldsymbol{n}
$$

and by applying Gauss's theorem, (2) can be localized

$$
\nabla \cdot \sigma+\rho \boldsymbol{f}=\rho \ddot{\boldsymbol{u}} .
$$

Using (3) and (4), the balance equation of angular momentum

$$
\frac{\mathrm{d}}{\mathrm{d} t} \int_{\mathcal{B}} \boldsymbol{x}_{\Delta} \times \rho \dot{\boldsymbol{u}} \mathrm{d} v=\int_{\mathcal{B}} \boldsymbol{x}_{\Delta} \times \rho \boldsymbol{f} \mathrm{d} v+\int_{\partial \mathcal{B}} \boldsymbol{x}_{\Delta} \times \boldsymbol{t} \mathrm{d} a
$$

with $\boldsymbol{x}_{\Delta}=\boldsymbol{x}-\boldsymbol{x}_{\text {ref }}$ denoting the difference vector to a fixed but otherwise arbitrary reference point $\boldsymbol{x}_{\text {ref }}$, reduces to the symmetry condition of the stress tensor

$$
\sigma=\sigma^{\mathrm{t}}
$$

\subsection{Electrical field equations}

General electro-magnetic problems are described in terms of Maxwell's equations. Being more specific, these are

$$
\begin{aligned}
& \text { Gauss's electric law } \quad \int_{\partial \mathcal{B}} \boldsymbol{d} \cdot \boldsymbol{n} \mathrm{d} a=\int_{\mathcal{B}} \rho_{\mathrm{f}} \mathrm{d} v \\
& \text { Gauss's magnetic law } \\
& \int_{\partial \mathcal{B}} \boldsymbol{b} \cdot \boldsymbol{n} \mathrm{d} a=0 \\
& \text { Faraday's law of induction } \\
& \int_{\partial \mathcal{A}} \boldsymbol{e} \cdot \mathrm{d} \boldsymbol{s}=-\frac{\mathrm{d}}{\mathrm{d} t} \int_{\mathcal{A}} \boldsymbol{b} \cdot \boldsymbol{n} \mathrm{d} \boldsymbol{a} \\
& \text { Ampère's circuital law } \\
& \int_{\partial \mathcal{A}} \boldsymbol{h} \cdot \mathrm{d} \boldsymbol{s}=\int_{\mathcal{A}} \boldsymbol{j} \cdot \boldsymbol{n} \mathrm{d} a+\frac{\mathrm{d}}{\mathrm{d} t} \int_{\mathcal{A}} \boldsymbol{d} \cdot \boldsymbol{n} \mathrm{d} a
\end{aligned}
$$


with the electric field vector $\boldsymbol{e}$, the dielectric displacement vector $\boldsymbol{d}$, the electric current density vector $\boldsymbol{j}$, the magnetic field vector $\boldsymbol{h}$, the magnetic flux density vector $\boldsymbol{b}$ and with the free charge density $\rho_{\mathrm{f}}$. Unlike $\partial \mathcal{B}$, area $\mathcal{A}$ needs not to be, and is generally not, closed. However, evaluating (7d) for a closed surface and inserting (7a) gives rise to the continuity equation for the electric current

$$
0=\int_{\partial \mathcal{B}} \boldsymbol{j} \cdot \boldsymbol{n} \mathrm{d} a+\frac{\mathrm{d}}{\mathrm{d} t} \int_{\mathcal{B}} \rho_{\mathrm{f}} \mathrm{d} v
$$

which is of particular importance for the developments to be presented in this contribution. Localizing the set of equations (7) by using Gauss's and Stokes' theorem yields

$$
\begin{array}{ll}
\text { Gauss's electric law } & \nabla \cdot \boldsymbol{d}=\rho_{\mathrm{f}} \\
\text { Gauss's magnetic law } & \nabla \cdot \boldsymbol{b}=0 \\
\text { Faraday's law of induction } & \nabla \times \boldsymbol{e}=-\dot{\boldsymbol{b}} \\
\text { Ampère's circuital law } & \nabla \times \boldsymbol{h}=\boldsymbol{j}+\dot{\boldsymbol{d}}
\end{array}
$$

and the localization of (8) results in

$$
\nabla \cdot \boldsymbol{j}+\dot{\rho}_{\mathrm{f}}=0
$$

Focusing on (quasi-)stationary electrical processes of conducting materials, Faraday's law (9c) reduces to

$$
\nabla \times \boldsymbol{e}=\mathbf{0}
$$

and the continuity equation for the electric current density is given by

$$
\nabla \cdot \boldsymbol{j}=0
$$

while the evaluation of Gauss's electric law (9a) yields the free charge density at every point of the continuum.

\subsection{Conservation of energy}

The balance equation of energy which governs the temperature evolution is given by

$$
\frac{\mathrm{d}}{\mathrm{dt}} \int_{\mathcal{B}} \rho e \mathrm{~d} v+\frac{\mathrm{d}}{\mathrm{dt}} \int_{\mathcal{B}} \frac{1}{2} \rho \dot{\boldsymbol{u}} \cdot \dot{\boldsymbol{u}} \mathrm{d} v=\int_{\mathcal{B}} \dot{\boldsymbol{u}} \cdot \rho \boldsymbol{f} \mathrm{d} v+\int_{\partial \mathcal{B}} \dot{\boldsymbol{u}} \cdot \boldsymbol{t} \mathrm{d} a+\int_{\mathcal{B}} \rho r \mathrm{~d} v-\int_{\partial \mathcal{B}} \boldsymbol{q} \cdot \boldsymbol{n} \mathrm{d} a+\int_{\mathcal{B}} \boldsymbol{j} \cdot \boldsymbol{e} \mathrm{d} v
$$

where, in addition to the mechanical working by body forces and surface tractions, thermal and electrical contributions are assumed to contribute to the (mass-specific) internal energy density $e$. The thermal contributions are given in terms of the heat source (per unit mass) $r$, which may, e.g., be related to chemical reactions, as well as in terms of the heat flux vector $\boldsymbol{q}$. The electrical contribution is given in terms of the electric current density vector $\boldsymbol{j}$ and its energetic dual, the electric field vector $\boldsymbol{e}$, see [5,8,25]. Localizing (13) by invoking Gauss's theorem and by making use of (3), (4) and (6) yields

$$
\rho \dot{e}=\sigma: \dot{\boldsymbol{\varepsilon}}+\rho r-\nabla \cdot \boldsymbol{q}+\boldsymbol{j} \cdot \boldsymbol{e}
$$

where the definition of the small strain deformation tensor

$$
\boldsymbol{\varepsilon}=\frac{1}{2}\left[\nabla \boldsymbol{u}+[\nabla \boldsymbol{u}]^{\mathrm{t}}\right]
$$

was used. 


\subsection{Dissipation inequality}

By introducing the (mass specific) entropy density $s$, the dissipation inequality characterizing the (thermo-)electro-mechanically coupled continuum reads

$$
\frac{\mathrm{d}}{\mathrm{dt}} \int_{\mathcal{B}} \rho s \mathrm{~d} v \geq \int_{\mathcal{B}} \frac{\rho r}{\theta} \mathrm{d} v-\int_{\partial \mathcal{B}} \frac{\boldsymbol{q} \cdot \boldsymbol{n}}{\theta} \mathrm{d} a .
$$

The localization of (16) by using Gauss's theorem yields

$$
\rho \dot{s} \geq \frac{\rho r}{\theta}-\frac{1}{\theta} \nabla \cdot \boldsymbol{q}+\frac{1}{\theta^{2}} \boldsymbol{q} \cdot \nabla \theta
$$

Moreover, introducing the (mass-specific) Helmholtz free energy density function $\psi$, making use of the convexconcave Legendre(-Fenchel) transformation

$$
\psi(\boldsymbol{\varepsilon}, \theta, \bullet)=\inf _{s}\{e(\boldsymbol{\varepsilon}, s, \bullet)-\theta s\}
$$

with the dual variables $\theta$ and $-s$ defined as

$$
\begin{aligned}
& \theta=\frac{\partial e}{\partial s} \\
& s=-\frac{\partial \psi}{\partial \theta}
\end{aligned}
$$

and inserting the local form of the balance equation of energy (14) yields

$$
\boldsymbol{\sigma}: \dot{\boldsymbol{\varepsilon}}-\rho[\dot{\psi}+s \dot{\theta}]-\frac{1}{\theta} \boldsymbol{q} \cdot \nabla \theta+\boldsymbol{j} \cdot \boldsymbol{e} \geq 0 .
$$

In addition to the well-established Clausius-Planck and Fourier inequalities, (20) poses restrictions on the specific form of the constitutive equation relating the electric field vector to the electric current density vector. In particular, it is observed that the (volume-specific) dissipation contribution $\boldsymbol{j} \cdot \boldsymbol{e}$ is associated with the electric current. The physical processes of electrodynamics, analyzed in the present contribution, are thus fundamentally different from the (possibly) reversible processes that occur in electro-active solids and that are studied in, e.g., [26].

\section{Multiscale modelling}

In computational multiscale formulations effective quantities at the macroscale are related to their counterparts at the microscale. This procedure is intrinsically based on averaging theorems for stresses, strains, electric current densities, and electric field strengths as discussed in Sect. 3.1. In addition, requiring micro-macro energy equivalence gives rise to the extended Hill-Mandel conditions presented in Sect. 3.2. Different sets of boundary conditions which a priori fulfil the averaging theorems and the extended Hill-Mandel conditions are finally established in Sect. 3.3. Due to the structural similarity of the continuity equation for the electric current density and the balance equation of linear momentum, and since the electric field is derived from a potential analogous to the derivation of the strain tensor from the displacement field, the ensuing derivations for the electrodynamic problem are similar to those of mechanical problems as documented in, e.g., [10,16,19-21]. Moreover, the structure of the underlying set of equations shares similarities with thermo-mechanically coupled problems as discussed in, e.g., [24] and with electro-mechanically coupled problems (for dielectric solids) as discussed in, e.g., [26]. Despite these structural similarities, there are significant differences between the present theory and the developments on electro-active solids presented in, e.g., [26]. The derivations presented in [26] are for example based on the assumptions of electrostatics while the present formulation relies on the fundamentals of electrodynamics such that different balance equations are solved, different field quantities occur, and different constitutive equations are postulated. In particular, the electric current is intrinsically dissipative as opposed to the (possibly) reversible processes in dielectric solids analyzed in [26]. 
In order to distinguish micro- and macroscale quantities, subscripts $\mathrm{m}$ (icro) and $\mathrm{M}(\mathrm{acro})$ are introduced. Moreover, quasi-static and quasi-stationary processes are assumed at the microscale. By additionally assuming negligible body forces, the set of balance equations to be solved at the microscale takes the form

$$
\begin{aligned}
& \nabla_{\mathrm{m}} \cdot \boldsymbol{\sigma}_{\mathrm{m}}=\mathbf{0}, \\
& \nabla_{\mathrm{m}} \cdot \boldsymbol{j}_{\mathrm{m}}=0,
\end{aligned}
$$

with Faraday's law (11) being accounted for by the introduction of an electric potential $\phi_{\mathrm{m}}$ such that

$$
\boldsymbol{e}_{\mathrm{m}}=-\nabla_{\mathrm{m}} \phi_{\mathrm{m}} \text {. }
$$

The assumptions that give rise to (21) are well-established for the mechanical problem and are for example discussed in [28].

\subsection{Averaging theorems}

In computational multiscale formulations, effective macroscopic quantities are defined as volume averages of their microscopic counterparts. For the evaluation of the generalized Hill-Mandel conditions, cf. Sect. 3.2, and from an implementation point of view it is moreover beneficial to rewrite the occurring volume integrals as surface integrals using Gauss's theorem. Invoking the identity

$$
\int_{\mathcal{B}_{\mathrm{m}}} \nabla_{\mathrm{m}} \bullet \mathrm{d} v=\int_{\mathcal{B}_{\mathrm{m}}}\left[\nabla_{\mathrm{m}} \bullet\right] \cdot \boldsymbol{I}^{\mathrm{t}} \mathrm{d} v=\int_{\mathcal{B}_{\mathrm{m}}} \nabla_{\mathrm{m}} \cdot[\bullet \otimes \boldsymbol{I}] \mathrm{d} v=\int_{\partial \mathcal{B}_{\mathrm{m}}} \bullet \otimes \boldsymbol{n}_{\mathrm{m}} \mathrm{d} a,
$$

and introducing the RVE-volume

$$
v_{\mathrm{m}}=\int_{\mathcal{B}_{\mathrm{m}}} \mathrm{d} v
$$

the effective macroscopic strain tensor may be specified in terms of boundary displacements, namely,

$$
\boldsymbol{\varepsilon}_{\mathrm{M}}=\frac{1}{v_{\mathrm{m}}} \int_{\mathcal{B}_{\mathrm{m}}} \boldsymbol{\varepsilon}_{\mathrm{m}} \mathrm{d} v=\frac{1}{v_{\mathrm{m}}} \int_{\mathcal{B}_{\mathrm{m}}} \frac{1}{2}\left[\nabla_{\mathrm{m}} \boldsymbol{u}_{\mathrm{m}}+\left[\nabla_{\mathrm{m}} \boldsymbol{u}_{\mathrm{m}}\right]^{\mathrm{t}}\right] \mathrm{d} v=\frac{1}{v_{\mathrm{m}}} \int_{\partial \mathcal{B}_{\mathrm{m}}} \frac{1}{2}\left[\boldsymbol{u}_{\mathrm{m}} \otimes \boldsymbol{n}_{\mathrm{m}}+\boldsymbol{n}_{\mathrm{m}} \otimes \boldsymbol{u}_{\mathrm{m}}\right] \mathrm{d} a
$$

Analogously, the effective macroscopic electric field vector is given by

$$
\boldsymbol{e}_{\mathrm{M}}=\frac{1}{v_{\mathrm{m}}} \int_{\mathcal{B}_{\mathrm{m}}} \boldsymbol{e}_{\mathrm{m}} \mathrm{d} v=\frac{1}{v_{\mathrm{m}}} \int_{\mathcal{B}_{\mathrm{m}}}-\nabla_{\mathrm{m}} \phi_{\mathrm{m}} \mathrm{d} v=\frac{1}{v_{\mathrm{m}}} \int_{\partial \mathcal{B}_{\mathrm{m}}}-\phi_{\mathrm{m}} \boldsymbol{n}_{\mathrm{m}} \mathrm{d} a .
$$

Moreover, the governing equation for the effective macroscopic stress tensor reads

$$
\begin{aligned}
& \sigma_{\mathrm{M}}=\frac{1}{v_{\mathrm{m}}} \int_{\mathcal{B}_{\mathrm{m}}} \boldsymbol{\sigma}_{\mathrm{m}} \mathrm{d} v=\frac{1}{v_{\mathrm{m}}} \int_{\mathcal{B}_{\mathrm{m}}} \boldsymbol{\sigma}_{\mathrm{m}} \cdot\left[\nabla_{\mathrm{m}} \boldsymbol{x}_{\mathrm{m}}\right]^{\mathrm{t}} \mathrm{d} v \\
& =\frac{1}{v_{\mathrm{m}}} \int_{\mathcal{B}_{\mathrm{m}}} \nabla_{\mathrm{m}} \cdot\left[\boldsymbol{\sigma}_{\mathrm{m}} \bar{\otimes} \boldsymbol{x}_{\mathrm{m}}\right] \mathrm{d} v-\frac{1}{v_{\mathrm{m}}} \int_{\mathcal{B}_{\mathrm{m}}} \nabla_{\mathrm{m}} \cdot\left[\boldsymbol{\sigma}_{\mathrm{m}}\right] \otimes \boldsymbol{x}_{\mathrm{m}} \mathrm{d} v \\
& =\frac{1}{v_{\mathrm{m}}} \int_{\partial \mathcal{B}_{\mathrm{m}}} \underbrace{\boldsymbol{\sigma}_{\mathrm{m}} \cdot \boldsymbol{n}_{\mathrm{m}}}_{=\boldsymbol{t}_{\mathrm{m}}} \otimes \boldsymbol{x}_{\mathrm{m}} \mathrm{d} a-\frac{1}{v_{\mathrm{m}}} \int_{\mathcal{B}_{\mathrm{m}}} \underbrace{\nabla_{\mathrm{m}} \cdot\left[\boldsymbol{\sigma}_{\mathrm{m}}\right]}_{=\mathbf{0}} \otimes \boldsymbol{x}_{\mathrm{m}} \mathrm{d} v
\end{aligned}
$$

and the one for the electric current density vector results in

$$
\begin{aligned}
\boldsymbol{j}_{\mathrm{M}}=\frac{1}{v_{\mathrm{m}}} \int_{\mathcal{B}_{\mathrm{m}}} \boldsymbol{j}_{\mathrm{m}} \mathrm{d} v & =\frac{1}{v_{\mathrm{m}}} \int_{\mathcal{B}_{\mathrm{m}}} \boldsymbol{j}_{\mathrm{m}} \cdot\left[\nabla_{\mathrm{m}} \boldsymbol{x}_{\mathrm{m}}\right]^{\mathrm{t}} \mathrm{d} v \\
& =\frac{1}{v_{\mathrm{m}}} \int_{\mathcal{B}_{\mathrm{m}}} \nabla_{\mathrm{m}} \cdot\left[\boldsymbol{x}_{\mathrm{m}} \otimes \boldsymbol{j}_{\mathrm{m}}\right] \mathrm{d} v-\frac{1}{v_{\mathrm{m}}} \int_{\mathcal{B}_{\mathrm{m}}} \nabla_{\mathrm{m}} \cdot\left[\boldsymbol{j}_{\mathrm{m}}\right] \boldsymbol{x}_{\mathrm{m}} \mathrm{d} v \\
& =\frac{1}{v_{\mathrm{m}}} \int_{\mathcal{B}_{\mathcal{B}_{\mathrm{m}}}} \boldsymbol{x}_{\mathrm{m}} \otimes \underbrace{\boldsymbol{j}_{\mathrm{m}} \cdot \boldsymbol{n}_{\mathrm{m}}}_{=i_{\mathrm{m}}} \mathrm{d} a-\frac{1}{v_{\mathrm{m}}} \int_{\mathcal{B}_{\mathrm{m}}}^{\nabla_{\mathrm{m}} \cdot\left[\boldsymbol{j}_{\mathrm{m}}\right]} \boldsymbol{x}_{=0}^{\boldsymbol{x}_{\mathrm{m}} \mathrm{d} v}
\end{aligned}
$$




\subsection{Hill-Mandel conditions}

The micro-macro energy equivalence condition, known in the literature as the Hill-Mandel condition, states that the volume average of the microscopic power in the RVE equals the local power on the macroscale. Focusing on the electro-mechanical problem introduced in Sect. 2 and denoting variations of field quantities - by $\delta \bullet$, the classic Hill-Mandel condition for the mechanical problem reads

$$
\begin{aligned}
\boldsymbol{\sigma}_{\mathrm{M}}: \delta \boldsymbol{\varepsilon}_{\mathrm{M}}=\frac{1}{v_{\mathrm{m}}} \int_{\mathcal{B}_{\mathrm{m}}} \boldsymbol{\sigma}_{\mathrm{m}}: \delta \boldsymbol{\varepsilon}_{\mathrm{m}} \mathrm{d} v & =\frac{1}{v_{\mathrm{m}}} \int_{\mathcal{B}_{\mathrm{m}}} \boldsymbol{\sigma}_{\mathrm{m}}: \nabla_{\mathrm{m}} \delta \boldsymbol{u}_{\mathrm{m}} \mathrm{d} v \\
& =\frac{1}{v_{\mathrm{m}}} \int_{\mathcal{B}_{\mathrm{m}}} \nabla_{\mathrm{m}} \cdot\left[\delta \boldsymbol{u}_{\mathrm{m}} \cdot \boldsymbol{\sigma}_{\mathrm{m}}\right] \mathrm{d} v-\frac{1}{v_{\mathrm{m}}} \int_{\partial \mathcal{B}_{\mathrm{m}}} \delta \boldsymbol{u}_{\mathrm{m}} \cdot\left[\nabla_{\mathrm{m}} \cdot \boldsymbol{\sigma}_{\mathrm{m}}\right] \mathrm{d} v \\
& =\frac{1}{v_{\mathrm{m}}} \int_{\partial \mathcal{B}_{\mathrm{m}}} \delta \boldsymbol{u}_{\mathrm{m}} \cdot \underbrace{\boldsymbol{\sigma}_{\mathrm{m}} \cdot \boldsymbol{n}_{\mathrm{m}}}_{=\boldsymbol{t}_{\mathrm{m}}} \mathrm{d} a-\frac{1}{v_{\mathrm{m}}} \int_{\mathcal{B}_{\mathrm{m}}} \delta \boldsymbol{u}_{\mathrm{m}} \cdot \underbrace{\nabla_{\mathrm{m}} \cdot\left[\boldsymbol{\sigma}_{\mathrm{m}}\right]}_{=\mathbf{0}} \mathrm{d} v
\end{aligned}
$$

and the one for the electrical problem is given by

$$
\begin{aligned}
\boldsymbol{j}_{\mathrm{M}} \cdot \delta \boldsymbol{e}_{\mathrm{M}}=\frac{1}{v_{\mathrm{m}}} \int_{\mathcal{B}_{\mathrm{m}}} \boldsymbol{j}_{\mathrm{m}} \cdot \delta \boldsymbol{e}_{\mathrm{m}} \mathrm{d} v & =-\frac{1}{v_{\mathrm{m}}} \int_{\mathcal{B}_{\mathrm{m}}} \boldsymbol{j}_{\mathrm{m}} \cdot \nabla_{\mathrm{m}} \delta \phi_{\mathrm{m}} \mathrm{d} v \\
& =-\frac{1}{v_{\mathrm{m}}} \int_{\mathcal{B}_{\mathrm{m}}} \nabla_{\mathrm{m}} \cdot\left[\delta \phi_{\mathrm{m}} \boldsymbol{j}_{\mathrm{m}}\right] \mathrm{d} v+\frac{1}{v_{\mathrm{m}}} \int_{\mathcal{B}_{\mathrm{m}}} \delta \phi_{\mathrm{m}}\left[\nabla_{\mathrm{m}} \cdot \boldsymbol{j}_{\mathrm{m}}\right] \mathrm{d} v \\
& =-\frac{1}{v_{\mathrm{m}}} \int_{\partial \mathcal{B}_{\mathrm{m}}} \delta \phi_{\mathrm{m}} \underbrace{\boldsymbol{j}_{\mathrm{m}} \cdot \boldsymbol{n}_{\mathrm{m}}}_{=i_{\mathrm{m}}} \mathrm{d} a+\frac{1}{v_{\mathrm{m}}} \int_{\mathcal{B}_{\mathrm{m}}} \delta \phi_{\mathrm{m}} \underbrace{\nabla_{\mathrm{m}} \cdot\left[\boldsymbol{j}_{\mathrm{m}}\right]}_{=0} \mathrm{~d} v
\end{aligned}
$$

Comprehensive discussions of the Hill-Mandel conditions for thermo-mechanically coupled problems, which are not in the focus of the present contribution, are presented in, e.g., $[6,28,29]$.

\subsection{Boundary conditions}

Different types of boundary conditions for the microstructural representative volume element can be defined which ensure that the Hill-Mandel conditions and the averaging theorems are fulfilled a priori. In the following, the classic three types of boundary conditions, i.e., affine and periodic as well as uniform flux boundary conditions, are briefly recapitulated for mechanical problems and the extension to electrical problems is shown.

\subsection{Affine boundary conditions}

Based on the macroscopic deformation state, affine displacement boundary conditions for the representative volume element are given by

$$
\boldsymbol{u}_{\mathrm{m}}=\boldsymbol{\varepsilon}_{\mathrm{M}} \cdot \boldsymbol{x}_{\mathrm{m}} \text { on } \partial \mathcal{B}_{\mathrm{m}}
$$

Analogously, taking into account the macroscopic electric potential and the macroscopic electric field strength, affine boundary conditions for the electric potential are defined as

$$
\phi_{\mathrm{m}}=\phi_{\mathrm{M}}-\boldsymbol{e}_{\mathrm{M}} \cdot \boldsymbol{x}_{\mathrm{m}} \quad \text { on } \partial \mathcal{B}_{\mathrm{m}} .
$$

The particular form (32) has been chosen in accordance with thermo-mechanical multiscale formulations as discussed in $[2,6]$. However, in contrast to the temperature, the electric potential is assumed to enter the constitutive equations only in terms of its gradient, i.e., the electric field vector, such that alternative definitions that do not account for $\phi_{\mathrm{M}}$ are possible.

The boundary conditions (31) and (32) a priori fulfil the averaging theorem for the strain tensor (25) and for the electric field vector (26) as well as the mechanical and the electrical Hill-Mandel condition, (29) 
respectively (30). More specifically speaking, by evaluating (25) for the boundary displacements given by (31) one arrives at

$$
\begin{aligned}
\frac{1}{v_{\mathrm{m}}} \int_{\mathcal{B}_{\mathrm{m}}} \boldsymbol{\varepsilon}_{\mathrm{m}} \mathrm{d} v=\frac{1}{v_{\mathrm{m}}} \int_{\partial \mathcal{B}_{\mathrm{m}}}\left[\left[\boldsymbol{\varepsilon}_{\mathrm{M}} \cdot \boldsymbol{x}_{\mathrm{m}}\right] \otimes \boldsymbol{n}_{\mathrm{m}}\right]^{\mathrm{sym}} \mathrm{d} a & =\frac{1}{v_{\mathrm{m}}}\left[\boldsymbol{\varepsilon}_{\mathrm{M}} \cdot \int_{\partial \mathcal{B}_{\mathrm{m}}} \boldsymbol{x}_{\mathrm{m}} \otimes \boldsymbol{I} \cdot \boldsymbol{n}_{\mathrm{m}} \mathrm{d} a\right]^{\mathrm{sym}} \\
& =\frac{1}{v_{\mathrm{m}}}\left[\boldsymbol{\varepsilon}_{\mathrm{M}} \cdot \int_{\mathcal{B}_{\mathrm{m}}} \nabla_{\mathrm{m}} \cdot\left[\boldsymbol{x}_{\mathrm{m}} \otimes \boldsymbol{I}\right] \mathrm{d} v\right]^{\mathrm{sym}}=\boldsymbol{\varepsilon}_{\mathrm{M}}
\end{aligned}
$$

and by inserting (32) into (26) at

$$
\begin{aligned}
\frac{1}{v_{\mathrm{m}}} \int_{\mathcal{B}_{\mathrm{m}}} \boldsymbol{e}_{\mathrm{m}} \mathrm{d} v=\frac{1}{v_{\mathrm{m}}} \int_{\partial \mathcal{B}_{\mathrm{m}}}-\left[\phi_{\mathrm{M}}-\boldsymbol{e}_{\mathrm{M}} \cdot \boldsymbol{x}_{\mathrm{m}}\right] \boldsymbol{n}_{\mathrm{m}} \mathrm{d} a & =\frac{1}{v_{\mathrm{m}}} \int_{\partial \mathcal{B}_{\mathrm{m}}}-\left[\phi_{\mathrm{M}}-\boldsymbol{e}_{\mathrm{M}} \cdot \boldsymbol{x}_{\mathrm{m}}\right] \boldsymbol{I} \cdot \boldsymbol{n}_{\mathrm{m}} \mathrm{d} a \\
& =\frac{1}{v_{\mathrm{m}}} \int_{\mathcal{B}_{\mathrm{m}}} \nabla_{\mathrm{m}} \cdot\left[-\left[\phi_{\mathrm{M}}-\boldsymbol{e}_{\mathrm{M}} \cdot \boldsymbol{x}_{\mathrm{m}}\right] \boldsymbol{I}\right] \mathrm{d} v=\boldsymbol{e}_{\mathrm{M}} .
\end{aligned}
$$

By additionally inserting (32) into the Hill-Mandel condition for the mechanical problem (29) and by making use of (27) one finds

$$
\frac{1}{v_{\mathrm{m}}} \int_{\mathcal{B}_{\mathrm{m}}} \boldsymbol{\sigma}_{\mathrm{m}}: \delta \boldsymbol{\varepsilon}_{\mathrm{m}} \mathrm{d} v=\frac{1}{v_{\mathrm{m}}} \int_{\partial \mathcal{B}_{\mathrm{m}}} \boldsymbol{t}_{\mathrm{m}} \cdot\left[\delta \boldsymbol{\varepsilon}_{\mathrm{M}} \cdot \boldsymbol{x}_{\mathrm{m}}\right] \mathrm{d} a=\frac{1}{v_{\mathrm{m}}} \int_{\partial \mathcal{B}_{\mathrm{m}}} \boldsymbol{t}_{\mathrm{m}} \otimes \boldsymbol{x}_{\mathrm{m}} \mathrm{d} a: \delta \boldsymbol{\varepsilon}_{\mathrm{M}}=\boldsymbol{\sigma}_{\mathrm{M}}: \delta \boldsymbol{\varepsilon}_{\mathrm{M}} .
$$

Likewise, the evaluation of (30) for (32), using (28), yields

$$
\frac{1}{v_{\mathrm{m}}} \int_{\mathcal{B}_{\mathrm{m}}} \boldsymbol{j}_{\mathrm{m}} \cdot \delta \boldsymbol{e}_{\mathrm{m}} \mathrm{d} v=-\frac{1}{v_{\mathrm{m}}} \int_{\partial \mathcal{B}_{\mathrm{m}}} i_{\mathrm{m}}\left[\delta \phi_{\mathrm{M}}-\delta \boldsymbol{e}_{\mathrm{M}} \cdot \boldsymbol{x}_{\mathrm{m}}\right] \mathrm{d} a=\frac{1}{v_{\mathrm{m}}} \int_{\partial \mathcal{B}_{\mathrm{m}}} i_{\mathrm{m}} \boldsymbol{x}_{\mathrm{m}} \mathrm{d} a \cdot \delta \boldsymbol{e}_{\mathrm{M}}=\boldsymbol{j}_{\mathrm{M}} \cdot \delta \boldsymbol{e}_{\mathrm{M}}
$$

\subsection{Periodic boundary conditions}

Indicating quantities at two opposing parts of the boundary, $\partial \mathcal{B}_{\mathrm{m}}^{+}$and $\partial \mathcal{B}_{\mathrm{m}}^{-}$, with superscripts $\bullet^{+}$and $\bullet^{-}$, the periodicity conditions for the mechanical problem are given by

$$
\begin{aligned}
\boldsymbol{u}_{\mathrm{m}}^{+}-\boldsymbol{u}_{\mathrm{m}}^{-} & =\boldsymbol{\varepsilon}_{\mathrm{M}} \cdot\left[\boldsymbol{x}_{\mathrm{m}}^{+}-\boldsymbol{x}_{\mathrm{m}}^{-}\right] \text {on } \partial \mathcal{B}_{\mathrm{m}}, \\
\boldsymbol{t}_{\mathrm{m}}^{+} & =-\boldsymbol{t}_{\mathrm{m}}^{-} \text {on } \partial \mathcal{B}_{\mathrm{m}},
\end{aligned}
$$

the ones for the electrical problem read

$$
\begin{aligned}
\phi_{\mathrm{m}}^{+}-\phi_{\mathrm{m}}^{-} & =-\boldsymbol{e}_{\mathrm{M}} \cdot\left[\boldsymbol{x}_{\mathrm{m}}^{+}-\boldsymbol{x}_{\mathrm{m}}^{-}\right] \text {on } \partial \mathcal{B}_{\mathrm{m}}, \\
i_{\mathrm{m}}^{+} & =-i_{\mathrm{m}}^{-} \text {on } \partial \mathcal{B}_{\mathrm{m}},
\end{aligned}
$$

and the classic geometric constraint for the unit outward surface normal vectors is stated as

$$
\boldsymbol{n}_{\mathrm{m}}^{+}=-\boldsymbol{n}_{\mathrm{m}}^{-} \text {on } \partial \mathcal{B}_{\mathrm{m}} .
$$

The set of equations (37) and (38) implies periodicity of the displacements and of the electric potential as well as anti-periodicity of the tractions and of the electric current densities.

Inserting (37a) into the averaging theorem for the strain tensor (25), using (39) and applying Gauss's theorem in the same way as in (33), yields

$$
\begin{aligned}
\frac{1}{v_{\mathrm{m}}} \int_{\mathcal{B}_{\mathrm{m}}} \boldsymbol{\varepsilon}_{\mathrm{m}} \mathrm{d} v=\frac{1}{v_{\mathrm{m}}}\left[\int_{\partial \mathcal{B}_{\mathrm{m}}^{+}} \boldsymbol{u}_{\mathrm{m}}^{+} \otimes \boldsymbol{n}_{\mathrm{m}}^{+} \mathrm{d} a+\int_{\partial \mathcal{B}_{\mathrm{m}}^{-}} \boldsymbol{u}_{\mathrm{m}}^{-} \otimes \boldsymbol{n}_{\mathrm{m}}^{-} \mathrm{d} a\right]^{\mathrm{sym}} & =\frac{1}{v_{\mathrm{m}}}\left[\int_{\partial \mathcal{B}_{\mathrm{m}}^{+}} \boldsymbol{\varepsilon}_{\mathrm{M}} \cdot\left[\boldsymbol{x}_{\mathrm{m}}^{+}-\boldsymbol{x}_{\mathrm{m}}^{-}\right] \otimes \boldsymbol{n}_{\mathrm{m}}^{+} \mathrm{d} a\right]^{\mathrm{sym}} \\
& =\frac{1}{v_{\mathrm{m}}}\left[\boldsymbol{\varepsilon}_{\mathrm{M}} \cdot \int_{\partial \mathcal{B}_{\mathrm{m}}} \boldsymbol{x}_{\mathrm{m}} \otimes \boldsymbol{I} \cdot \boldsymbol{n}_{\mathrm{m}} \mathrm{d} a\right]^{\mathrm{sym}}=\boldsymbol{\varepsilon}_{\mathrm{M}} .
\end{aligned}
$$


Analogously, by inserting (38a) into (26) one finds

$$
\begin{aligned}
\frac{1}{v_{\mathrm{m}}} \int_{\mathcal{B}_{\mathrm{m}}} \boldsymbol{e}_{\mathrm{m}} \mathrm{d} v=-\frac{1}{v_{\mathrm{m}}}\left[\int_{\partial \mathcal{B}_{\mathrm{m}}^{+}} \phi_{\mathrm{m}}^{+} \boldsymbol{n}_{\mathrm{m}}^{+} \mathrm{d} a+\int_{\partial \mathcal{B}_{\mathrm{m}}^{-}} \phi_{\mathrm{m}}^{-} \boldsymbol{n}_{\mathrm{m}}^{-} \mathrm{d} a\right] & =\frac{1}{v_{\mathrm{m}}}\left[\int_{\partial \mathcal{B}_{\mathrm{m}}^{+}} \boldsymbol{e}_{\mathrm{M}} \cdot\left[\boldsymbol{x}_{\mathrm{m}}^{+}-\boldsymbol{x}_{\mathrm{m}}^{-}\right] \otimes \boldsymbol{n}_{\mathrm{m}}^{+} \mathrm{d} a\right] \\
& =\frac{1}{v_{\mathrm{m}}}\left[\boldsymbol{e}_{\mathrm{M}} \cdot \int_{\partial \mathcal{B}_{\mathrm{m}}} \boldsymbol{x}_{\mathrm{m}} \otimes \boldsymbol{I} \cdot \boldsymbol{n}_{\mathrm{m}} \mathrm{d} a\right]=\boldsymbol{e}_{\mathrm{M}} .
\end{aligned}
$$

Evaluating the mechanical and electrical Hill-Mandel conditions, (29) and (30), for the periodic boundary conditions (37) and (38) eventually yields

$$
\begin{aligned}
\frac{1}{v_{\mathrm{m}}} \int_{\mathcal{B}_{\mathrm{m}}} \boldsymbol{\sigma}_{\mathrm{m}}: \delta \boldsymbol{\varepsilon}_{\mathrm{m}} \mathrm{d} v=\frac{1}{v_{\mathrm{m}}}\left[\int_{\partial \mathcal{B}_{\mathrm{m}}^{+}} \boldsymbol{t}_{\mathrm{m}}^{+} \cdot \delta \boldsymbol{u}_{\mathrm{m}}^{+} \mathrm{d} a+\int_{\partial \mathcal{B}_{\mathrm{m}}^{-}} \boldsymbol{t}_{\mathrm{m}}^{-} \cdot \delta \boldsymbol{u}_{\mathrm{m}}^{-} \mathrm{d} a\right] & =\frac{1}{v_{\mathrm{m}}} \int_{\partial \mathcal{B}_{\mathrm{m}}^{+}} \boldsymbol{t}_{\mathrm{m}}^{+} \cdot\left[\delta \boldsymbol{\varepsilon}_{\mathrm{M}} \cdot\left[\boldsymbol{x}_{\mathrm{m}}^{+}-\boldsymbol{x}_{\mathrm{m}}^{-}\right]\right] \mathrm{d} a \\
& =\frac{1}{v_{\mathrm{m}}} \int_{\partial \mathcal{B}_{\mathrm{m}}} \boldsymbol{t}_{\mathrm{m}} \otimes \boldsymbol{x}_{\mathrm{m}} \mathrm{d} a: \delta \boldsymbol{\varepsilon}_{\mathrm{M}}=\boldsymbol{\sigma}_{\mathrm{M}}: \delta \boldsymbol{\varepsilon}_{\mathrm{M}}
\end{aligned}
$$

and

$$
\begin{aligned}
\frac{1}{v_{\mathrm{m}}} \int_{\mathcal{B}_{\mathrm{m}}} \boldsymbol{j}_{\mathrm{m}} \cdot \delta \boldsymbol{e}_{\mathrm{m}} \mathrm{d} v=-\frac{1}{v_{\mathrm{m}}}\left[\int_{\partial \mathcal{B}_{\mathrm{m}}^{+}} i_{\mathrm{m}}^{+} \delta \phi_{\mathrm{m}}^{+} \mathrm{d} a+\int_{\partial \mathcal{B}_{\mathrm{m}}^{-}} i_{\mathrm{m}}^{-} \delta \phi_{\mathrm{m}}^{-} \mathrm{d} a\right] & =\frac{1}{v_{\mathrm{m}}} \int_{\partial \mathcal{B}_{\mathrm{m}}^{+}} i_{\mathrm{m}}^{+}\left[\delta \boldsymbol{e}_{\mathrm{M}} \cdot\left[\boldsymbol{x}_{\mathrm{m}}^{+}-\boldsymbol{x}_{\mathrm{m}}^{-}\right]\right] \mathrm{d} a \\
& =\frac{1}{v_{\mathrm{m}}} \int_{\partial \mathcal{B}_{\mathrm{m}}} i_{\mathrm{m}} \boldsymbol{x}_{\mathrm{m}} \mathrm{d} a \cdot \delta \boldsymbol{e}_{\mathrm{M}}=\boldsymbol{j}_{\mathrm{M}} \cdot \delta \boldsymbol{e}_{\mathrm{M}}
\end{aligned}
$$

\subsection{Uniform flux boundary conditions}

As opposed to affine and periodic boundary conditions, Neumann-type boundary conditions in terms of tractions and electric current densities are prescribed at the boundary of the representative volume element when uniform flux boundary conditions are applied. For the mechanical boundary value problem these are given by

$$
\boldsymbol{t}_{\mathrm{m}}=\boldsymbol{\sigma}_{\mathrm{M}} \cdot \boldsymbol{n}_{\mathrm{m}} \quad \text { on } \partial \mathcal{B}_{\mathrm{m}},
$$

and those for the electrical problem read

$$
i_{\mathrm{m}}=\boldsymbol{j}_{\mathrm{M}} \cdot \boldsymbol{n}_{\mathrm{m}} \text { on } \partial \mathcal{B}_{\mathrm{m}} .
$$

The equality of the macroscopic stress tensor $\sigma_{M}$ and of the averaged microscopic stress tensor for uniform traction boundary conditions follows immediately by inserting (44) into (27), to be specific

$$
\frac{1}{v_{\mathrm{m}}} \int_{\mathcal{B}_{\mathrm{m}}} \boldsymbol{\sigma}_{\mathrm{m}} \mathrm{d} v=\frac{1}{v_{\mathrm{m}}} \int_{\partial \mathcal{B}_{\mathrm{m}}} \boldsymbol{\sigma}_{\mathrm{M}} \cdot \boldsymbol{n}_{\mathrm{m}} \otimes \boldsymbol{x}_{\mathrm{m}} \mathrm{d} a-\frac{1}{v_{\mathrm{m}}} \int_{\mathcal{B}_{\mathrm{m}}} \nabla_{\mathrm{m}} \cdot\left[\boldsymbol{\sigma}_{\mathrm{M}}\right] \otimes \boldsymbol{x}_{\mathrm{m}} \mathrm{d} v=\frac{1}{v_{\mathrm{m}}} \int_{\mathcal{B}_{\mathrm{m}}} \boldsymbol{\sigma}_{\mathrm{M}} \mathrm{d} v=\boldsymbol{\sigma}_{\mathrm{M}}
$$

Analogously, the insertion of (45) into (28) yields

$$
\frac{1}{v_{\mathrm{m}}} \int_{\mathcal{B}_{\mathrm{m}}} \boldsymbol{j}_{\mathrm{m}} \mathrm{d} v=\frac{1}{v_{\mathrm{m}}} \int_{\partial \mathcal{B}_{\mathrm{m}}} \boldsymbol{x}_{\mathrm{m}} \otimes \boldsymbol{j}_{\mathrm{M}} \cdot \boldsymbol{n}_{\mathrm{m}} \mathrm{d} a-\frac{1}{v_{\mathrm{m}}} \int_{\mathcal{B}_{\mathrm{m}}} \nabla_{\mathrm{m}} \cdot\left[\boldsymbol{j}_{\mathrm{M}}\right] \boldsymbol{x}_{\mathrm{m}} \mathrm{d} v=\frac{1}{v_{\mathrm{m}}} \int_{\mathcal{B}_{\mathrm{m}}} \boldsymbol{j}_{\mathrm{M}} \mathrm{d} v=\boldsymbol{j}_{\mathrm{M}}
$$

The evaluation of the Hill-Mandel conditions (29) and (30) furthermore results in

$$
\frac{1}{v_{\mathrm{m}}} \int_{\mathcal{B}_{\mathrm{m}}} \boldsymbol{\sigma}_{\mathrm{m}}: \delta \boldsymbol{\varepsilon}_{\mathrm{m}} \mathrm{d} v=\frac{1}{v_{\mathrm{m}}} \int_{\partial \mathcal{B}_{\mathrm{m}}} \delta \boldsymbol{u}_{\mathrm{m}} \cdot \boldsymbol{\sigma}_{\mathrm{M}} \cdot \boldsymbol{n}_{\mathrm{m}} \mathrm{d} v=\boldsymbol{\sigma}_{\mathrm{M}}: \frac{1}{v_{\mathrm{m}}}\left[\int_{\partial \mathcal{B}_{\mathrm{m}}} \delta \boldsymbol{u}_{\mathrm{m}} \otimes \boldsymbol{n}_{\mathrm{m}} \mathrm{d} v\right]^{\mathrm{sym}}=\boldsymbol{\sigma}_{\mathrm{M}}: \delta \boldsymbol{\varepsilon}_{\mathrm{M}}
$$

and in

$$
\frac{1}{v_{\mathrm{m}}} \int_{\mathcal{B}_{\mathrm{m}}} \boldsymbol{j}_{\mathrm{m}} \cdot \delta \boldsymbol{e}_{\mathrm{m}} \mathrm{d} v=-\frac{1}{v_{\mathrm{m}}} \int_{\partial \mathcal{B}_{\mathrm{m}}} \delta \phi_{\mathrm{m}} \boldsymbol{j}_{\mathrm{M}} \cdot \boldsymbol{n}_{\mathrm{m}} \mathrm{d} a=\boldsymbol{j}_{\mathrm{M}} \cdot\left[-\frac{1}{v_{\mathrm{m}}} \int_{\partial \mathcal{B}_{\mathrm{m}}} \delta \phi_{\mathrm{m}} \boldsymbol{n}_{\mathrm{m}} \mathrm{d} a\right]=\boldsymbol{j}_{\mathrm{M}} \cdot \delta \boldsymbol{e}_{\mathrm{M}}
$$

where (25) and (26) were used. 


\section{Finite element implementation}

This section focuses on the finite element implementation of the electro-mechanically coupled multiscale formulation proposed in Sect. 3. In particular, the homogenization of effective macroscopic stresses and electric current densities is discussed in Sect. 4.1, and the corresponding algorithmic tangent stiffness tensors are derived in Sect. 4.2. The ensuing derivations rely on and are direct extensions of well-established computational homogenization procedures. For a detailed elaboration of these fundamentals, the reader is referred to, e.g., the pioneering work [19] which focuses on the purely mechanical case. The extension to thermo-mechanically coupled multi-field problems is moreover discussed in [24,28] and electro-mechanically coupled problems (of dielectric materials) are for instance studied in $[13,14]$.

The subsequent derivations apply to affine and periodic boundary conditions. In the case of periodic boundary conditions, however, the generalized stiffness matrix $\mathbf{K}_{\mathrm{m}}$ and the generalized reaction force vector $f_{\mathrm{m}}$ must be substituted by the reduced generalized stiffness matrix $\mathbf{K}_{\mathrm{m}}^{*}$ and by the reduced generalized reaction force vector $f_{\mathrm{m}}^{*}$ that result from the application of linear constraints when enforcing (anti-)periodicity. Specifically speaking, the system

$$
\mathbf{K}_{\mathrm{m}} \cdot \Delta \mathcal{X}_{\mathrm{m}}=\Delta \boldsymbol{f}_{\mathrm{m}}
$$

is rewritten in each iteration step in the form

$$
\mathbf{K}_{\mathrm{m}}^{*} \cdot \Delta \mathcal{X}_{\mathrm{mi}}=\Delta \boldsymbol{f}_{\mathrm{m}}^{*}
$$

with

$$
\mathbf{K}_{\mathrm{m}}^{*}=\mathcal{T}^{\mathrm{t}} \cdot \mathbf{K}_{\mathrm{m}} \cdot \mathcal{T}, \quad \boldsymbol{f}_{\mathrm{m}}^{*}=\mathcal{T}^{\mathrm{t}} \cdot \boldsymbol{f}_{\mathrm{m}}, \quad\left[\begin{array}{c}
\mathcal{X}_{\mathrm{mi}} \\
\mathcal{X}_{\mathrm{md}}
\end{array}\right]=\mathcal{T} \cdot \mathcal{X}_{\mathrm{mi}}
$$

using the transformation matrix $\mathcal{T}$ that relates dependent $\mathcal{X}_{\text {md }}$ and independent $\mathcal{X}_{\text {mi }}$ degrees of freedom, see [30].

\subsection{Homogenization}

Following standard procedure, the discrete version of (27) is given by

$$
\boldsymbol{\sigma}_{\mathrm{M}}=\frac{1}{v_{\mathrm{m}}} \int_{\partial \mathcal{B}_{\mathrm{m}}} \boldsymbol{t}_{\mathrm{m}} \otimes \boldsymbol{x}_{\mathrm{m}} \mathrm{d} a \approx \frac{1}{v_{\mathrm{m}}} \sum_{i=1}^{\mathrm{n}_{\mathrm{pn}}}{ }^{(i)} \boldsymbol{f}_{\mathrm{m}}^{\boldsymbol{u}} \otimes{ }^{(i)} \boldsymbol{x}_{\mathrm{m}},
$$

with $\mathrm{n}_{\mathrm{pn}}$ denoting the number of nodes where the corresponding degrees of freedom are prescribed, and with ${ }^{(i)} \boldsymbol{x}_{\mathrm{m}}$ and ${ }^{(i)} \boldsymbol{f}_{\mathrm{m}}^{\boldsymbol{u}}$ denoting the position vector and the reaction force vector of node $i$. Analogously, the discrete version of (28) is given by

$$
\boldsymbol{j}_{\mathrm{M}}=\frac{1}{v_{\mathrm{m}}} \int_{\partial \mathcal{B}_{\mathrm{m}}} i_{\mathrm{m}} \boldsymbol{x}_{\mathrm{m}} \mathrm{d} a \approx \frac{1}{v_{\mathrm{m}}} \sum_{i=1}^{\mathrm{n}_{\mathrm{pn}}}{ }^{(i)} f_{\mathrm{m}}^{\phi(i)} \boldsymbol{x}_{\mathrm{m}},
$$

with ${ }^{(i)} f_{\mathrm{m}}^{\phi}$ denoting the generalized reaction force of node $i$, corresponding to the electrical problem.

\subsection{Generalized algorithmic tangent stiffness tensors}

In order to derive generalized algorithmic tangent stiffness operators, changes in the generalized reaction forces $\Delta \boldsymbol{f}_{\mathrm{mp}}$ due to perturbations of the prescribed degrees of freedom $\Delta \mathcal{X}_{\mathrm{mp}}$ are studied. To this end, the partitioned system of equations

$$
\left[\begin{array}{ll}
\mathbf{K}_{\mathrm{mpp}} & \mathbf{K}_{\mathrm{mpf}} \\
\mathbf{K}_{\mathrm{mfp}} & \mathbf{K}_{\mathrm{mff}}
\end{array}\right] \cdot\left[\begin{array}{l}
\Delta \mathcal{X}_{\mathrm{mp}} \\
\Delta \mathcal{X}_{\mathrm{mf}}
\end{array}\right]=\left[\begin{array}{c}
\Delta \boldsymbol{f}_{\mathrm{mp}} \\
\mathbf{0}
\end{array}\right]
$$


with subscripts $\bullet_{\mathrm{p}}$ and $\bullet_{\text {f }}$ referring to prescribed and free degrees of freedom is considered. Solving the second set of equations for $\Delta \mathcal{X}_{\mathrm{mf}}$ and inserting the result into the first set of equations results in

$$
\widehat{\mathbf{K}}_{\mathrm{m}} \cdot \Delta \mathcal{X}_{\mathrm{mp}}=\Delta \boldsymbol{f}_{\mathrm{mp}} \text { with } \widehat{\mathbf{K}}_{\mathrm{m}}=\mathbf{K}_{\mathrm{mpp}}-\mathbf{K}_{\mathrm{mpf}} \cdot \mathbf{K}_{\mathrm{mff}}^{-1} \cdot \mathbf{K}_{\mathrm{mfp}}
$$

For affine as well as for periodic boundary conditions, the kinematic relations

$$
\Delta^{(j)} \boldsymbol{u}_{\mathrm{mp}}=\Delta \boldsymbol{\varepsilon}_{\mathrm{M}} \cdot{ }^{(j)} \boldsymbol{x}_{\mathrm{m}}
$$

and

$$
\Delta^{(j)} \phi_{\mathrm{mp}}=\Delta \phi_{\mathrm{M}}-\Delta \boldsymbol{e}_{\mathrm{M}} \cdot{ }^{(j)} \boldsymbol{x}_{\mathrm{m}}
$$

hold for nodes where the respective degrees of freedom are prescribed. In the case of affine boundary conditions, these are the boundary nodes while the set consists of three corner nodes in a two-dimensional setting, respectively, of four corner nodes in a three-dimensional setting, when periodic boundary conditions are applied. By partitioning (56) into mechanical and electrical contributions and after inserting the kinematic relations (57) and (58), it is observed that changes in the generalized reaction force vectors at the microscale due to changes in $\boldsymbol{\varepsilon}_{\mathrm{M}}$ and $\boldsymbol{e}_{\mathrm{M}}$ are given by

$$
\begin{aligned}
\Delta^{(i)} \boldsymbol{f}_{\mathrm{m}}^{\boldsymbol{u}} & =\sum_{j=1}^{\mathrm{n}_{\mathrm{pn}}}{ }^{(i j)} \widehat{\mathbf{K}}_{\mathrm{m}}^{\boldsymbol{u} \boldsymbol{u}} \cdot \Delta^{(j)} \boldsymbol{u}_{\mathrm{mp}}+\sum_{j=1}^{\mathrm{n}_{\mathrm{pn}}}{ }^{(i j)} \widehat{\mathbf{K}}_{\mathrm{m}}^{\boldsymbol{u \phi}} \Delta^{(j)} \phi_{\mathrm{mp}} \\
& =\sum_{j=1}^{\mathrm{n}_{\mathrm{pn}}}{ }^{(i j)} \widehat{\mathbf{K}}_{\mathrm{m}}^{\boldsymbol{u} \boldsymbol{u}} \cdot \Delta \boldsymbol{\varepsilon}_{\mathrm{M}} \cdot{ }^{(j)} \boldsymbol{x}_{\mathrm{m}}-\sum_{j=1}^{\mathrm{n}_{\mathrm{pn}}}{ }^{(i j)} \widehat{\mathbf{K}}_{\mathrm{m}}^{\boldsymbol{u \phi}} \otimes \Delta \boldsymbol{e}_{\mathrm{M}} \cdot{ }^{(j)} \boldsymbol{x}_{\mathrm{m}}
\end{aligned}
$$

and

$$
\begin{aligned}
\Delta^{(i)} f_{\mathrm{m}}^{\phi} & =\sum_{j=1}^{\mathrm{n}_{\mathrm{pn}}}{ }^{(i j)} \widehat{\mathbf{K}}_{\mathrm{m}}^{\phi \boldsymbol{u}} \cdot \Delta^{(j)} \boldsymbol{u}_{\mathrm{m} \mathrm{p}} \quad+\sum_{j=1}^{{ }^{\mathrm{n}_{\mathrm{pn}}}}{ }^{(i j)} \widehat{\mathbf{K}}_{\mathrm{m}}^{\phi \phi} \Delta^{(j)} \phi_{\mathrm{mp}} \\
& =\sum_{j=1}^{\mathrm{n}_{\mathrm{pn}}}{ }^{(i j)} \widehat{\mathbf{K}}_{\mathrm{m}}^{\phi \boldsymbol{u}} \cdot \Delta \boldsymbol{\varepsilon}_{\mathrm{M}} \cdot{ }^{(j)} \boldsymbol{x}_{\mathrm{m}}-\sum_{j=1}^{{ }^{\mathrm{n}_{\mathrm{pn}}}}{ }^{(i j)} \widehat{\mathbf{K}}_{\mathrm{m}}^{\phi \phi} \Delta \boldsymbol{e}_{\mathrm{M}} \cdot{ }^{(j)} \boldsymbol{x}_{\mathrm{m}}
\end{aligned}
$$

In accordance with (53) and (54), superscripts $\bullet^{\boldsymbol{u}}$ and $\bullet \boldsymbol{\phi}$ refer to mechanical and electrical field equations and fields, respectively. Moreover, it is assumed that the electric potential enters the field equations and constitutive equations only via its gradient, i.e., via the electric field vector, such that changes in $\phi_{\mathrm{M}}$ are neglected in (59), (60) and in the following. A dependence of the constitutive equations on the electric potential would lead to problems analogous to those of the thermal case, as discussed in [29].

Eventually, the insertion of (59) into (53) gives rise to the definition of the macroscale algorithmic tangent stiffness tensors

$$
\Delta \boldsymbol{\sigma}_{\mathrm{M}} \approx \underbrace{\left[\frac{1}{v_{\mathrm{m}}} \sum_{i=1}^{\mathrm{n}_{\mathrm{pn}}} \sum_{j=1}^{\mathrm{n}_{\mathrm{pn}}}{ }^{(i j)} \widehat{\mathbf{K}}_{\mathrm{m}}^{\boldsymbol{u} \boldsymbol{u}} \bar{\otimes}\left[{ }^{(i)} \boldsymbol{x}_{\mathrm{m}} \otimes{ }^{(j)} \boldsymbol{x}_{\mathrm{m}}\right]\right]}_{=\frac{\mathrm{d} \boldsymbol{\sigma}_{\mathrm{M}}}{\mathrm{d} \boldsymbol{\varepsilon}_{\mathrm{M}}}}: \Delta \boldsymbol{\varepsilon}_{\mathrm{M}}+\underbrace{\left[-\frac{1}{v_{\mathrm{m}}} \sum_{i=1}^{\mathrm{n}_{\mathrm{pn}}} \sum_{j=1}^{\mathrm{n}_{\mathrm{pn}}}{ }^{(i j)} \widehat{\mathbf{K}}_{\mathrm{m}}^{\boldsymbol{u} \boldsymbol{\phi}} \otimes{ }^{(i)} \boldsymbol{x}_{\mathrm{m}} \otimes{ }^{(j)} \boldsymbol{x}_{\mathrm{m}}\right]}_{=\frac{\mathrm{d} \boldsymbol{\sigma}_{\mathrm{M}}}{\mathrm{d} \boldsymbol{e}_{\mathrm{M}}}} \cdot \Delta \boldsymbol{e}_{\mathrm{M}} .
$$

Likewise, inserting (60) into (54) yields

$$
\Delta \boldsymbol{j}_{\mathrm{M}} \approx \underbrace{\left[\frac{1}{v_{\mathrm{m}}} \sum_{i=1}^{\mathrm{n}_{\mathrm{pn}}} \sum_{j=1}^{\mathrm{n}_{\mathrm{pn}}}{ }^{(i)} \boldsymbol{x}_{\mathrm{m}} \otimes{ }^{(i j)} \widehat{\mathbf{K}}_{\mathrm{m}}^{\phi \boldsymbol{u}} \otimes{ }^{(j)} \boldsymbol{x}_{\mathrm{m}}\right]}_{=\frac{\mathrm{d} \boldsymbol{j}_{\mathrm{M}}}{\mathrm{d} \boldsymbol{\varepsilon}_{\mathrm{M}}}}: \Delta \boldsymbol{\varepsilon}_{\mathrm{M}}+\underbrace{\left[-\frac{1}{v_{\mathrm{m}}} \sum_{i=1}^{\mathrm{n}_{\mathrm{pn}}} \sum_{j=1}^{\mathrm{n}_{\mathrm{pn}}}{ }^{(i j)} \widehat{\mathbf{K}}_{\mathrm{m}}^{\phi \phi}{ }^{(i)} \boldsymbol{x}_{\mathrm{m}} \otimes{ }^{(j)} \boldsymbol{x}_{\mathrm{m}}\right]}_{=\frac{\mathrm{d} \boldsymbol{j}_{\mathrm{M}}}{\mathrm{d} \boldsymbol{e}_{\mathrm{M}}}} \cdot \Delta \boldsymbol{e}_{\mathrm{M}} .
$$




\section{Representative simulation results}

This section focuses on the application of the proposed electro-mechanical multiscale formulation to two- and three dimensional quasi-static and quasi-stationary boundary value problems. To this end, the material model that is used at the microscale is briefly discussed in Sect. 5.1. In a second step, the calculation of effective electrical conductivity tensors for two-dimensional microstructures is discussed in Sect. 5.2, before tension test samples with different microstructures are subjected to detailed three-dimensional multiscale analyses in Sect. 5.3.

\subsection{Microscale material models}

On the microscale, constitutive models that characterise the material behavior need to be developed subject to the restrictions posed by the dissipation inequality (20). Moreover, the specific form of the constitutive equations is decisive for the coupling between the electrical and the mechanical field equations. Different physical effects that result in an explicit and implicit coupling of the field equations were outlined in Sect. 1 and motivate the present contribution. However, since the focus of the present work lies on the fundamentals of the electro-mechanically coupled computational multiscale formulation and not on the development of a particular microscale material model, we restrict ourselves to isotropic, linear electrical and mechanical microscale material models and postpone the analysis of more elaborated material models to future works. Thus, for the particular material model considered, the electrical and the mechanical field equations remain uncoupled. Moreover, superscripts $\bullet_{\mathrm{m}}$ referring to the microscale are neglected in the following for the sake of brevity.

By introducing the volume-specific Helmholtz free energy density function $\Psi(\boldsymbol{\varepsilon}, \theta, \bullet)=\rho \psi(\boldsymbol{\varepsilon}, \theta, \bullet)$, the evaluation of the dissipation inequality (20) yields the standard definition of the stress tensor

$$
\sigma=\frac{\partial \Psi}{\partial \boldsymbol{\varepsilon}}
$$

In conjunction with the specific quadratic form of the volume specific Helmholtz free energy density function

$$
\widetilde{\Psi}(\varepsilon)=\frac{1}{2} \varepsilon: \mathbf{E}: \varepsilon
$$

with $\mathbf{E}$ denoting the fourth-order elasticity tensor

$$
\mathbf{E}=\frac{E v}{[1+v][1-2 v]} \boldsymbol{I} \otimes \boldsymbol{I}+\frac{E}{2[1+v]}[\boldsymbol{I} \bar{\otimes} \boldsymbol{I}+\boldsymbol{I} \otimes \underline{I}]
$$

and with $E$ and $v$ denoting the Young's modulus and Poisson's ratio, the evaluation of (63) results in

$$
\sigma=\mathbf{E}: \varepsilon
$$

In addition, by introducing the positive definite electrical conductivity tensor

$$
\mathcal{S}=\kappa \boldsymbol{I},
$$

with the idealized scalar-valued conductivity $\kappa$, a suitable choice for the electric current density vector that is in accordance with (20) is given by

$$
j=\mathcal{S} \cdot e
$$


Table 1 Material parameters of an idealized material used in the simulations

\begin{tabular}{lll}
\hline$E$ & $v$ & $\kappa$ \\
$130,000 \mathrm{~N} / \mathrm{mm}^{2}$ & 0.35 & $58,100 \mathrm{~A} /[\mathrm{Vmm}]$ \\
\hline
\end{tabular}

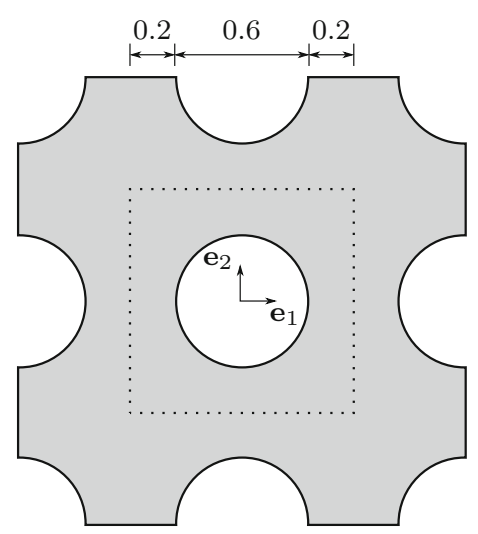

(a) circular

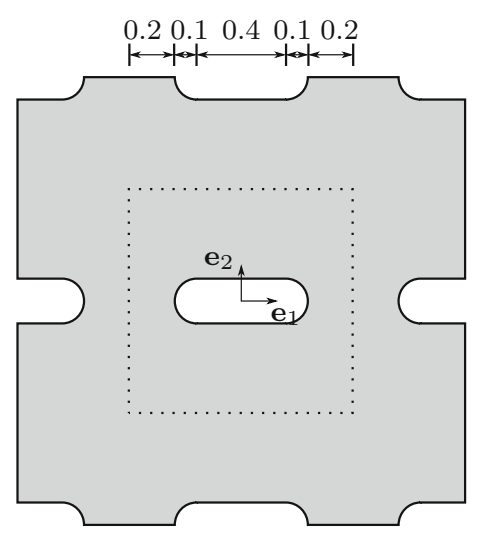

(b) groove-shaped

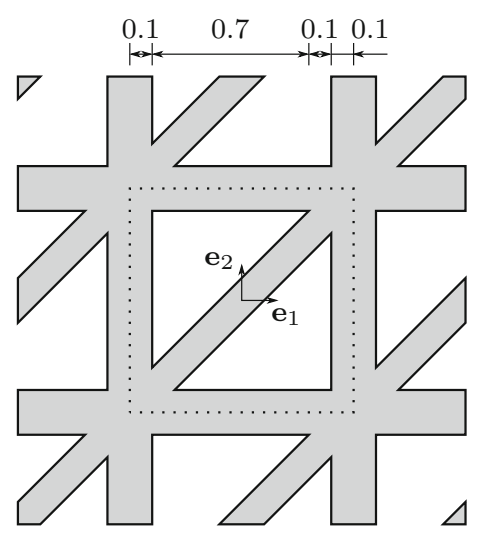

(c) diagonal

Fig. 2 Sketch of different two-dimensional microstructures analyzed with the electro-mechanical multiscale finite element formulation

\subsection{Two-dimensional representative simulations}

This section focuses on the application of the proposed electro-mechanical multiscale formulation in a twodimensional plane strain setting. To this end, idealized material parameters of copper are assumed at the microscale, and effective macroscopic conductivity tensors are exemplarily calculated for different microstructures. The material parameters are summarized in Table 1 and three different microstructures to be analyzed are depicted in Fig. 2.

The microstructures are assumed to be periodic and the representative volume elements which form the basis for the finite element simulations are indicated by dashed lines. For the sake of clarity, only the dimensions in $\mathbf{e}_{1}$-direction are provided. However, since symmetry with respect to the $\mathbf{e}_{1}$ - and $\mathbf{e}_{2}$-direction or to the space diagonals is assumed, the remaining dimensions follow implicitly.

The application of the homogenization scheme with periodic boundary conditions as discussed in Sect. 4 to the microstructures depicted in Fig. 2 yields the macroscopic conductivity tensors

$$
\begin{aligned}
{\left[\mathcal{S}_{\mathrm{M}}\right]_{i j}^{\mathrm{cir}} } & =\left[\begin{array}{ll}
0.5592 & 0.0000 \\
0.0000 & 0.5592
\end{array}\right] \kappa \\
{\left[\mathcal{S}_{\mathrm{M}}\right]_{i j}^{\text {gro }} } & =\left[\begin{array}{ll}
0.8575 & 0.0000 \\
0.0000 & 0.6445
\end{array}\right] \kappa \\
{\left[\mathcal{S}_{\mathrm{M}}\right]_{i j}^{\mathrm{dia}} } & =\left[\begin{array}{ll}
0.3126 & 0.0716 \\
0.0716 & 0.3126
\end{array}\right] \kappa
\end{aligned}
$$

with superscripts referring to the respective microstructure. Comparing the effective macroscopic conductivity tensor of a material with circular voids (69a) with the conductivity tensor of an idealized material (67), it is observed that the effective macroscopic constitutive response remains isotropic whereas a reduction in the conductivity of about $45 \%$ is observed. In contrast, the effective macroscopic conductivity tensor (69b) that results from a microstructure with a groove-shaped void as depicted in Fig. $2 b$ is significantly anisotropic. However, the principal material axes are aligned with the $\mathbf{e}_{1}$ - and $\mathbf{e}_{2}$-coordinate axes such that the conductivity tensor is in diagonal form. In the case of the microstructure of diagonal type depicted in Fig. 2c, the $\mathbf{e}_{1}$ - and $\mathbf{e}_{2}$ coordinate axes are not aligned with the principal material axes. Thus, the effective macroscopic conductivity tensor (69c) is not in diagonal form but features off-diagonal components.

The previous examples demonstrate the applicability of the electro-mechanical multiscale formulation to extract effective macroscopic conductivity tensors for given microstructures. This approach may be interesting 


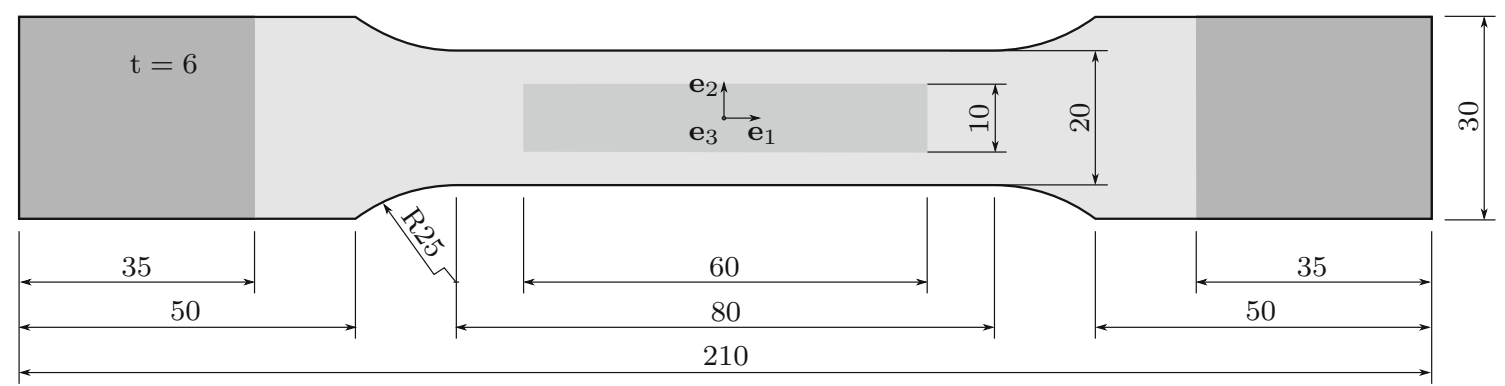

Fig. 3 Tension test specimen of type E according to DIN 50125, see [7], used in the electro-mechanical multiscale finite element simulations. Dark grey color indicates regions where Dirichlet boundary conditions are applied. The light grey colored $60 \mathrm{~mm} \times 10 \mathrm{~mm} \times 6 \mathrm{~mm}$ region in the center of the specimen resembles material featuring microscale imperfections. All dimensions are given in $\mathrm{mm}$

Table 2 Multiscale finite element simulations of tension test samples with different microstructures according to Fig. 3. The difference in the axial reaction force and the difference in the electric current for prescribed displacements and prescribed electric potential differences is provided

\begin{tabular}{|c|c|c|c|c|}
\hline & \multicolumn{2}{|l|}{ Electric current } & \multicolumn{2}{|c|}{ Electric current difference } \\
\hline Voltage & Perfect material & Material with voids & Absolute & Relative \\
\hline \multirow[t]{2}{*}{$0.1 \mathrm{mV}$} & $5458 \mathrm{~mA}$ & $5368 \mathrm{~mA}$ & $-90 \mathrm{~mA}$ & $-1.65 \%$ \\
\hline & \multicolumn{2}{|c|}{ Axial reaction force } & \multicolumn{2}{|c|}{ Axial reaction force difference } \\
\hline Elongation & Perfect material & Material with voids & Absolute & Relative \\
\hline $0.21 \mathrm{~mm}$ & $25.63 \mathrm{kN}$ & $25.12 \mathrm{kN}$ & $0.51 \mathrm{kN}$ & $-1.99 \%$ \\
\hline
\end{tabular}

for electrical engineering applications to developed tailored microstructures with specific electrical properties. Moreover, regarding electro-mechanical coupling, changes in effective macroscopic conductivities that are accessible via non-destructive testing methods may be related to microcracks that are induced by mechanical loadings.

\subsection{Three-dimensional representative simulations}

As an extension of the two-dimensional sample boundary value problems discussed in Sect. 5.2 to a threedimensional setting, this section focuses on the effective macroscopic responses of tensile test specimens with different material microstructures. The tensile test specimen of type $\mathrm{E}$ that serves as the basis for the simulations is sketched in Fig. 3, with supports at the left and right boundaries being indicated by dark grey color. Specifically speaking, homogeneous Dirichlet boundary conditions for the electrical and mechanical fields are assumed at the top and bottom surface nodes of the left boundary. Likewise, homogeneous Dirichlet boundary conditions for the mechanical field are applied in $\mathbf{e}_{2}$ - and $\mathbf{e}_{3}$-direction at the top and bottom nodes of the right boundary, while both the displacement in $\mathbf{e}_{1}$-direction as well as the electric potential take values different from zero, see Table 2.

In order to study the influence of different microstructures on the effective electrical and mechanical properties at the macroscale, simulation results of a tensile test specimen with a perfect microstructure as depicted in Fig. $4 \mathrm{~b}$ are compared to those of a tensile test specimen with an imperfect microstructure. Specifically speaking, imperfections on the microscale are assumed in the light grey colored $60 \mathrm{~mm} \times 10 \mathrm{~mm} \times 6 \mathrm{~mm}$ region in the center of the specimen and are given in terms of a spherical void with a radius of 0.23 RVE-edge length as depicted in Fig. 4d. In accordance with the two-dimensional simulation results presented in Sect. 5.2, isotropic, linear constitutive relations are assumed for the mechanical and electric problem at each material point at the microscale. Furthermore, the same set of material parameters as in the two-dimensional simulations is used, see Table 1, and periodic boundary conditions are applied at the microscale.

The distributions of the electric current density at the micro- and macroscale are provided in Fig. 4 for tensile test samples with perfect and imperfect microstructures. By comparing the distributions of the electric current density at the macroscale, Fig. 4a, c, it is observed that the material imperfection manifests itself in a reduction of the electric current density in the center region of the specimen. This effect can be explained by 


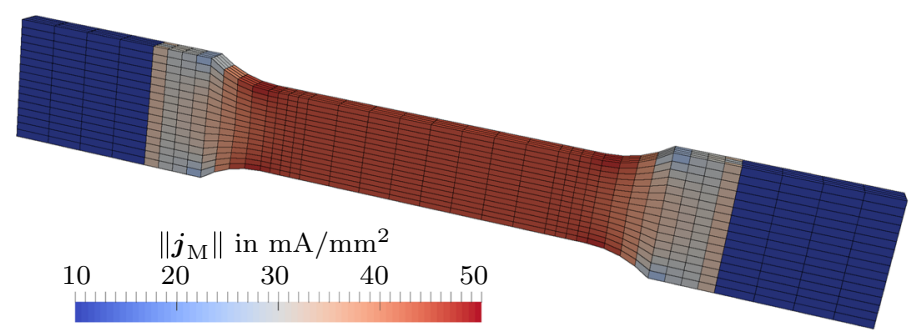

(a) macroscale, perfect material

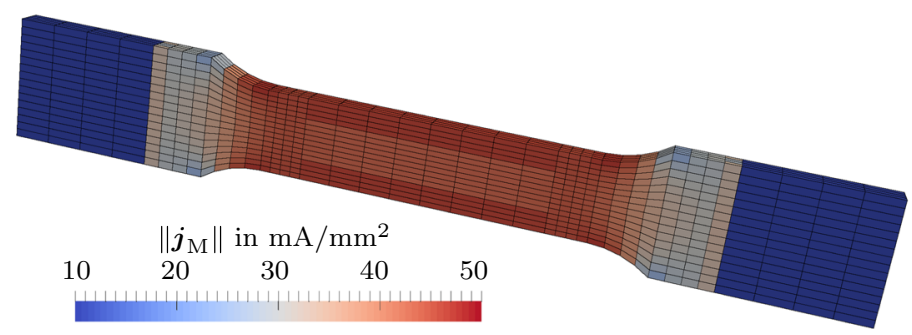

(c) macroscale, material with voids

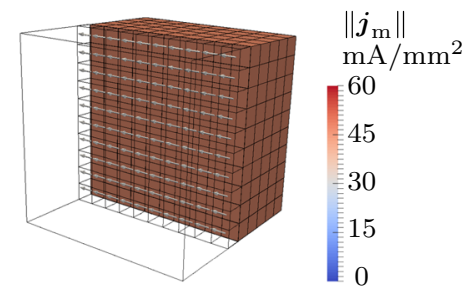

(b) microscale, perfect material

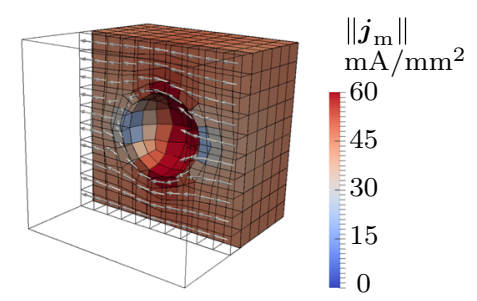

(d) microscale, material with voids

Fig. 4 Electro-mechanical multiscale finite element simulation results. The electric current density distributions on the macroscale (a, c) and on the microscale (b, d), near the center of the specimen, are depicted. The simulation results for a tensile test specimen with a perfect microstructure $(\mathbf{a}, \mathbf{b})$ differ significantly from the ones with an imperfect microstructure that contains voids (c, d). Gray-colored arrows indicate the electric current density vector. Only one half of the representative volume element is shown

the microscale simulation results depicted in Fig. $4 \mathrm{~b}$, d which show that the material imperfection acts as an obstacle for the electric current. In addition, the force in $\mathbf{e}_{1}$-direction and the electric current that both occur as generalized reaction forces dual to the applied displacement and to the applied electric potential difference, respectively, are summarized in Table 2. In accordance with the simulation results of Fig. 4, the effective macroscopic electric current takes smaller values in the case of a material with an imperfect microstructure. The same observation is made for the reaction force in $\mathbf{e}_{2}$-direction.

\section{Closure}

Summary Motivated by advances in flexible electronic technologies and by the desire to develop non-destructive testing methods, an electro-mechanical multiscale formulation for conductors is proposed in this contribution. After a brief recapitulation of the governing set of electro-mechanical field equations, scale-bridging relations for the electro-mechanical problem are proposed. In addition, a finite element implementation of the electromechanical multiscale formulation is discussed, and different types of boundary condition are shown to a priori fulfil the extended Hill-Mandel energy equivalence conditions. Several numerical examples are studied in two- and three-dimensional settings to show the applicability of the proposed formulation. In particular, the calculation of effective macroscopic conductivity tensors for given two-dimensional microstructures is discussed, which is of interest for electrical engineering applications when developing tailored microstructures with specific electrical properties. Moreover, the study of tensile test samples with different microstructures, which are subjected to electro-mechanical boundary conditions, reveals the influence of the microstructure on the effective macroscopic electrical and mechanical material properties. The interrelation of the latter is of special interest in the development of nondestructive testing methods, and is in the focus of future works.

Outlook Multiscale approaches are based on an accurate simulation of processes that occur at a lower scale. For this reason, more elaborated electro-mechanically coupled material models are to be developed and to be used at the microscale, e.g., in order to account for the influence of mechanically induced microcracks on the electrical conductivity. Against this background and with tailored microscale material models being beyond the scope of the present contribution, assume for now a deformation-dependent conductivity tensor of the form

$$
\widetilde{\mathcal{S}}=\exp \left(-\frac{\widetilde{\Psi}}{\kappa_{*}}\right) \kappa \boldsymbol{I}
$$




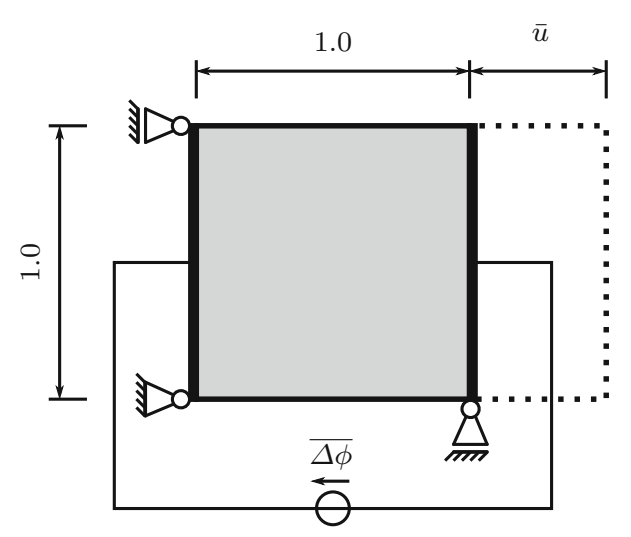

Fig. 5 Macroscale boundary value problem for the study of nonlinear electro-mechanical coupling effects

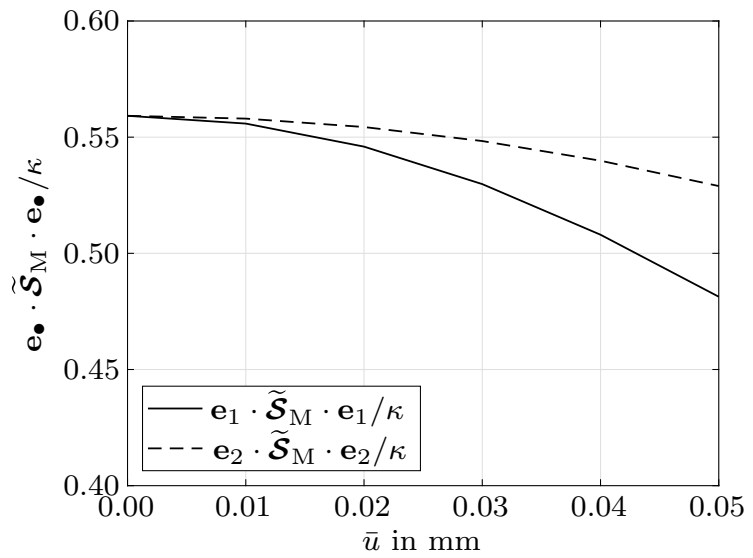

(a) effective macroscopic conductivity

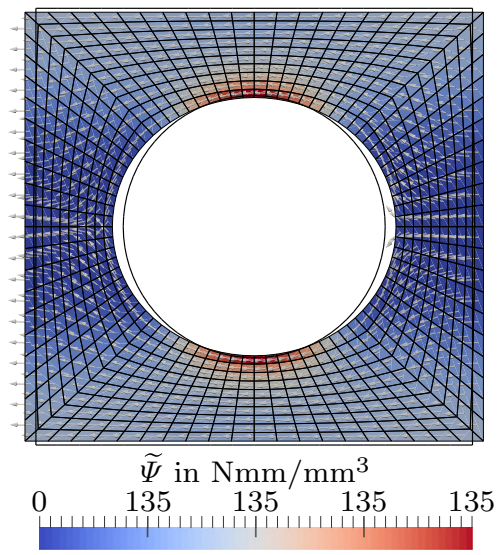

(b) microscale deformation

Fig. 6 a Electrical conductivity as a function of prescribed macro-displacement $\bar{u}$. b Deformation of the microscale RVE. The Helmholtz free energy density distribution for load state $\bar{u}=0.05 \mathrm{~mm}$ is shown on the deformed configuration. In addition, the electric current density vector field for an applied potential difference of $\overline{\Delta \phi}=0.1 \mathrm{mV}$ is indicated by grey-colored arrows and the outline of the undeformed reference configuration is provided in terms of thick black lines

with

$$
\begin{aligned}
& \frac{\mathrm{d} \boldsymbol{j}}{\mathrm{d} \boldsymbol{e}}=\widetilde{\mathcal{S}} \\
& \frac{\mathrm{d} \boldsymbol{j}}{\mathrm{d} \boldsymbol{\varepsilon}}=-\frac{\kappa}{\kappa_{*}} \exp \left(-\frac{\widetilde{\Psi}}{\kappa_{*}}\right) \boldsymbol{e} \otimes \sigma,
\end{aligned}
$$

and with material parameter $\kappa_{*}$ controlling the decrease in electrical conductivity with increasing deformation. The particular form of (70) is based on the idea that damage evolution is intrinsically energy-driven and that cracks represent obstacles for the electric current. Of course, the simplified format considered here does not include any history variables.

To study the latter material model in a multiscale environment, a two-dimensional plane strain one-element test as depicted in Fig. 5 is analyzed, with a periodic microstructure according to Fig. 2a being assumed. For the mechanical sub-problem the material model (63)-(66) is adopted. Moreover, the material parameters are chosen according to Table 1 and $\kappa_{*}=0.01 E$ is assumed.

The decrease in the 11- and 22-coefficient of the effective macroscopic conductivity tensor as a function of deformation in terms of the prescribed macroscale displacement $\bar{u}$ is provided in Fig. 6a, with a significantly different decrease of the electrical conductivity in the two spatial directions being observed. The latter finding can be explained by the inhomogeneous microscale deformation as exemplarily shown in Fig. 6b. For the analyzed load state, the severe deformation zones that occur at the top and bottom of the circular void result in a significant decrease of the electrical conductivity in $\mathbf{e}_{1}$-direction. 
Remark 1 Motivated by the introductory example in Sect. 1, geometry effects may be emulated in a small strain setting by introducing the deformation dependent conductivity tensor

$$
\widehat{\mathcal{S}}=\kappa \frac{\boldsymbol{e} \cdot[\boldsymbol{I}+\operatorname{tr}(\boldsymbol{\varepsilon}) \boldsymbol{I}-\boldsymbol{\varepsilon}] \cdot \boldsymbol{e}}{\boldsymbol{e} \cdot[\boldsymbol{I}+\boldsymbol{\varepsilon}] \cdot \boldsymbol{e}}
$$

The derivation of $\widehat{\mathcal{S}}$ is motivated by geometrically induced changes in resistance of an idealized wire according to (1). Additionally, linearized versions of the transport theorems for infinitesimal line elements

$$
[\boldsymbol{I}+\nabla \boldsymbol{u}] \cdot \mathrm{d} \boldsymbol{S}=\mathrm{d} \boldsymbol{s} \text { with } \boldsymbol{I}+\nabla \boldsymbol{u}=\boldsymbol{I}+\boldsymbol{\varepsilon}+\frac{1}{2}\left[\nabla \boldsymbol{u}-[\nabla \boldsymbol{u}]^{\mathrm{t}}\right]
$$

and surface elements

$$
\operatorname{cof}(\boldsymbol{I}+\nabla \boldsymbol{u}) \cdot \boldsymbol{N} \mathrm{d} A=\boldsymbol{n} \mathrm{d} a \quad \text { with } \quad \operatorname{cof}(\boldsymbol{I}+\nabla \boldsymbol{u}) \approx \boldsymbol{I}+\operatorname{tr}(\boldsymbol{\varepsilon}) \boldsymbol{I}-[\nabla \boldsymbol{u}]^{\mathrm{t}}
$$

are used, with referential and spatial representations being indicated by $\mathrm{d} S, \mathrm{~d} A, \boldsymbol{N}$, respectively, by $\mathrm{d} \boldsymbol{s}, \mathrm{d} a, \boldsymbol{n}$.

Acknowledgements Financial support from the German Research Foundation (DFG) via SFB/TR TRR 188 is gratefully acknowledged.

Funding Open access funding is provided by Project DEAL.

Open Access This article is licensed under a Creative Commons Attribution 4.0 International License, which permits use, sharing, adaptation, distribution and reproduction in any medium or format, as long as you give appropriate credit to the original author(s) and the source, provide a link to the Creative Commons licence, and indicate if changes were made. The images or other third party material in this article are included in the article's Creative Commons licence, unless indicated otherwise in a credit line to the material. If material is not included in the article's Creative Commons licence and your intended use is not permitted by statutory regulation or exceeds the permitted use, you will need to obtain permission directly from the copyright holder. To view a copy of this licence, visit http://creativecommons.org/licenses/by/4.0/.

\section{Compliance with ethical standards}

Conflict of interest On behalf of all authors, the corresponding author states that there is no conflict of interest.

\section{References}

1. Axisa, F., Schmitt, P.M., Gehin, C., Delhomme, G., McAdams, E., Dittmar, A.: Flexible technologies and smart clothing for citizen medicine, home healthcare, and disease prevention. IEEE Trans. Inf. Technol. Biomed. 9(3), 325-336 (2005)

2. Berthelsen, R., Menzel, A.: Computational homogenisation of thermo-viscoplastic composites: Large strain formulation and weak micro-periodicity. Comput. Methods Appl. Mech. Eng. 348, 575-603 (2019)

3. Chen, Y., Au, J., Kazlas, P., Ritenour, A., Gates, H., McCreary, M.: Flexible active-matrix electronic ink display. Nature 423(6936), 136 (2003)

4. Cordill, M.J., Glushko, O., Kreith, J., Marx, V.M., Kirchlechner, C.: Measuring electro-mechanical properties of thin films on polymer substrates. Microelectron. Eng. 137, 96-100 (2015)

5. Eringen, A.C.: Mechanics of Continua. Robert E. Krieger Publishing Co, New York (1980)

6. Berthelsen, R., Denzer, R., Oppermann, P., Menzel, A.: Computational homogenisation for thermoviscoplasticity: Application to thermally sprayed coatings. Comput. Mech. 60(5), 739-766 (2017)

7. DIN 50125:2016-12, Prüfung metallischer Werkstoffe - Zugprobe

8. Eringen, A.C., Maugin, G.A.: Electrodynamics of Continua I: Foundations and Solid Media. Springer-Verlag New York Inc, New York (1990)

9. Fangye, Y.F., Miska, N., Balzani, D.: Automated simulation of voxel-based microstructures based on enhanced finite cell approach. Arch. Appl. Mech. 90, 2255-2273 (2020)

10. Feyel, F., Chaboche, J.L.: $\mathrm{FE}^{2}$ multiscale approach for modelling the elastoviscoplastic behaviour of long fibre $\mathrm{SiC} / \mathrm{Ti}$ composite materials. Comput. Methods Appl. Mech. Eng. 183(3), 309-330 (2000)

11. Gu, T., Castelnau, O., Forest, S., Hervé-Luanco, E., Lecouturier, F., Proudhon, H., Thilly, L.: Multiscale modeling of the elastic behavior of architectured and nanostructured $\mathrm{Cu}-\mathrm{Nb}$ composite wires. Int. J. Solids Struct. 121, 148-162 (2017)

12. Jänicke, R., Steeb, H.: Minimal loading conditions for higher-order numerical homogenisation schemes. Arch. Appl. Mech. 82(8), 1075-1088 (2012)

13. Keip, M.A., Steinmann, P., Schröder, J.: Two-scale computational homogenization of electro-elasticity at finite strains. Comput. Methods Appl. Mech. Eng. 278, 62-79 (2014)

14. Khalaquzzaman, M., Xu, B.X., Ricker, S., Müller, R.: Computational homogenization of piezoelectric materials using FE2 to determine configurational forces. Technische Mechanik 32(1), 21-37 (2012) 
15. Kim, S., Kwon, H.J., Lee, S., Shim, H., Chun, Y., Choi, W., Kwack, J., Han, D., Song, M., Kim, S., Mohammadi, S., Kee, I., Lee, S.Y.: Low-power flexible organic light-emitting diode display device. Adv. Mater. 23(31), 3511-3516 (2011)

16. Kouznetsova, V.G., Brekelmans, W.A.M., Baaijens, F.P.T.: An approach to micro-macro modeling of heterogeneous materials. Comput. Mech. 27(1), 37-48 (2001)

17. Kouznetsova, V.G., Geers, M.G.D., Brekelmans, W.A.M.: Multi-scale constitutive modelling of heterogeneous materials with a gradient-enhanced computational homogenization scheme. Int. J. Numer. Meth. Eng. 54(8), 1235-1260 (2002)

18. Kouznetsova, V.G., Geers, M.G.D., Brekelmans, W.A.M.: Multi-scale second-order computational homogenization of multiphase materials: A nested finite element solution strategy. Comput. Methods Appl. Mech. Eng. 193(48), 5525-5550 (2004)

19. Miehe, C., Koch, A.: Computational micro-to-macro transitions of discretized microstructures undergoing small strains. Arch. Appl. Mech. 72(4-5), 300-317 (2002)

20. Miehe, C., Schotte, J., Schröder, J.: Computational micro-macro transitions and overall moduli in the analysis of polycrystals at large strains. Comput. Mater. Sci. 16(1), 372-382 (1999)

21. Miehe, C., Schröder, J., Schotte, J.: Computational homogenization analysis in finite plasticity. Simulation of texture development in polycrystalline materials. Comput. Methods Appl. Mech. Eng. 171(3), 387-418 (1999)

22. Mitsubayashi, K., Wakabayashi, Y., Murotomi, D., Yamada, T., Kawase, T., Iwagaki, S., Karube, I.: Wearable and flexible oxygen sensor for transcutaneous oxygen monitoring. Sens. Actuators Chem. 95(1), 373-377 (2003)

23. Ostoja-Starzewski, M.: Macrohomogeneity condition in dynamics of micropolar media. Arch. Appl. Mech. 81(7), 899-906 (2011)

24. Özdemir, I., Brekelmans, W.A.M., Geers, M.G.D.: FE ${ }^{2}$ computational homogenization for the thermo-mechanical analysis of heterogeneous solids. Comput. Methods Appl. Mech. Eng. 198(3), 602-613 (2008)

25. Santapuri, S.: Thermodynamic restrictions on linear reversible and irreversible thermo-electro-magneto-mechanical processes. Heliyon 2(10), e00164 (2016)

26. Schröder, J.: Derivation of the localization and homogenization conditions for electro-mechanically coupled problems. Comput. Mater. Sci. 46(3), 595-599 (2009)

27. Schröder, J., Balzani, D., Brands, D.: Approximation of random microstructures by periodic statistically similar representative volume elements based on lineal-path functions. Arch. Appl. Mech. 81(7), 975-997 (2011)

28. Sengupta, A., Papadopoulos, P., Taylor, R.L.: A multiscale finite element method for modeling fully coupled thermomechanical problems in solids. Int. J. Numer. Meth. Eng. 91(13), 1386-1405 (2012)

29. Temizer, İ., Wriggers, P.: Homogenization in finite thermoelasticity. J. Mech. Phys. Solids 59(2), 344-372 (2011)

30. Webb, J.P.: Imposing linear constraints in finite-element analysis. Commun. Appl. Numer. Methods 6(6), 471-475 (1990)

Publisher's Note Springer Nature remains neutral with regard to jurisdictional claims in published maps and institutional affiliations. 